\title{
Barriers to transport in aperiodically time-dependent two-dimensional velocity fields: Nekhoroshev's theorem and "Nearly Invariant" tori
}

\author{
S. Wiggins ${ }^{1}$ and A. M. Mancho ${ }^{2}$ \\ ${ }^{1}$ School of Mathematics, University of Bristol, Bristol BS8 1TW, UK \\ ${ }^{2}$ Instituto de Ciencias Matemáticas, CSIC-UAM-UC3M-UCM, C/Nicolás Cabrera 15, \\ Campus Cantoblanco UAM, 28049 Madrid, Spain \\ Correspondence to: S. Wiggins (s.wiggins@ bristol.ac.uk)
}

Received: 26 July 2013 - Revised: 12 December 2013 - Accepted: 17 December 2013 - Published: 4 February 2014

\begin{abstract}
In this paper we consider fluid transport in twodimensional flows from the dynamical systems point of view, with the focus on elliptic behaviour and aperiodic and finite time dependence. We give an overview of previous work on general nonautonomous and finite time vector fields with the purpose of bringing to the attention of those working on fluid transport from the dynamical systems point of view a body of work that is extremely relevant, but appears not to be so well known. We then focus on the Kolmogorov-Arnold-Moser (KAM) theorem and the Nekhoroshev theorem. While there is no finite time or aperiodically time-dependent version of the KAM theorem, the Nekhoroshev theorem, by its very nature, is a finite time result, but for a "very long" (i.e. exponentially long with respect to the size of the perturbation) time interval and provides a rigorous quantification of "nearly invariant tori" over this very long timescale. We discuss an aperiodically time-dependent version of the Nekhoroshev theorem due to Giorgilli and Zehnder (1992) (recently refined by Bounemoura, 2013 and Fortunati and Wiggins, 2013) which is directly relevant to fluid transport problems. We give a detailed discussion of issues associated with the applicability of the KAM and Nekhoroshev theorems in specific flows. Finally, we consider a specific example of an aperiodically time-dependent flow where we show that the results of the Nekhoroshev theorem hold.
\end{abstract}

\section{Introduction}

This paper is concerned with "Kolmogorov, Arnold, Moser (KAM) like behavior" in two-dimensional, incompressible, aperiodically time-dependent velocity fields over a finite time interval. We will explain what we mean by this phrase in the course of the introduction, and we will begin by noting that the motivation for this study comes from the dynamical systems approach to Lagrangian transport in fluid flows.

Let $\mathbf{r} \equiv(x, y)$ denote coordinates describing a twodimensional region. A fluid flow in this region is described by a velocity field, $\mathbf{v}(\mathbf{r}, t) \equiv\left(v_{x}(x, y, t), v_{y}(x, y, t)\right)$. The velocity field can be kinematically defined (i.e. constructed to describe certain observed features of the flow), dynamically defined (i.e. it is obtained as the solution of a set of partial differential equations that describe the dynamical evolution of the velocity field), or it could be obtained by observation (i.e. through remote sensing of some region of the ocean). In any case, at this point of the discussion, it is not important how one obtains the velocity field, but we assume that by some means we have obtained a velocity field. The equations describing the motion of fluid particles in the velocity field are given by:

$\dot{\mathbf{r}}=\mathbf{v}(\mathbf{r}, t)$

(neglecting molecular diffusion, or possibly the effect of neglected scales of motion, which would be the case if one were considering velocity fields obtained from partial differential equations that describe only certain length and time scales in the ocean). If the flow is incompressible the velocity field 
can be obtained from the derivatives of a scalar valued function $\psi(x, y, t)$, the stream function, as (see, e.g., Batchelor (1967)):

$\dot{x}=\frac{\partial \psi}{\partial y}(x, y, t)$,

$\dot{y}=-\frac{\partial \psi}{\partial x}(x, y, t)$.

Making the connection with the mathematical framework of dynamical systems theory, formally Eq. (2) has the form of Hamilton's canonical equations, where $\psi(x, y, t)$ plays the role of the Hamiltonian function and the corresponding phase space coordinates, $(x, y)$, are actually the physical space coordinates in which the fluid flow takes place. If $\psi(x, y, t)$ is periodic in the time, $t$, then it is standard in dynamical systems theory to study the structure of the trajectories of Eq. (2) by considering the (discrete) orbits of the associated Poincaré map, i.e. to view the continuous time trajectories of Eq. (2) at a sequence of discrete times, where the sequence of times is integer multiples of the (temporal) period of the stream function.

This "Hamiltonian dynamical systems" point of view generated a great deal of interest and further research starting in the early 1980s with the publication of Aref (1984). This occurred at the same time that "applied dynamical systems theory" was flowering as a topic of study across many disciplines in science and engineering. The growing availability of computational resources was giving rise to a large amount of "computational phenomenology" for twodimensional, area preserving maps (such as the standard map; see e.g. Meiss, 2008). Thus, armed with the point of view described in Aref (1984), one could "see" that phase space structures such as elliptic periodic orbits, hyperbolic periodic orbits and their stable and unstable manifolds, and KAM tori had an immediate interpretation in terms of "structures" in the flow influencing transport and mixing. In this way dynamical systems theory provided an analytical and computational meaning for the notion of "coherent structures" in fluid flows that was becoming a frequent observation in experiments due to advances in flow visualization capabilities (see e.g. Brown and Roshko, 1974). For example, transversely intersecting stable and unstable manifolds of hyperbolic periodic orbits give rise to "chaotic fluid particle trajectories" through the construction on Smale horseshoes, KAM tori trap regions of fluid (therefore preventing them from "mixing" with surrounding fluid), KAM tori are found surrounding elliptic periodic orbits (hence these are a "signature" of regions of unmixed fluid), and the intersecting stable and unstable manifolds give rise to "partial" barriers to transport and "lobe dynamics". This mathematical framework proved to be ideal for realizing the physical picture of mixing put forth earlier by Reynolds (1894), Eckart (1948), Danckwerts (1952), and Danckwerts (1953). Ottino et al. (1994) has described in detail the physical picture of mixing first described by Reynolds and how it had to await the proper mathematical framework, i.e. dynamical systems theory, before it could be analysed and exploited. Reviews of the dynamical systems approach to Lagrangian transport and mixing, mostly for two-dimensional, time-periodic incompressible flows, can be found in Aref and El Naschie (1994), Aref (2002), Acrivos et al. (1991), Babiano et al. (1994), Ottino (1989a, b), Wiggins (1992), Wiggins and Ottino (2004), and Sturman et al. (2006).

The new insights into transport and mixing obtained from the dynamical systems approach for two-dimensional, incompressible, time-periodic flows motivated efforts to extend this approach to more complex flow situations. Two possible extensions would be from two to three space dimensions and from periodic time dependence to a more general time dependence. Some motivation for these extensions came from the desire to use the dynamical systems approach to study Lagrangian transport in the ocean and atmosphere, starting in the early 1990s. Such flows will generally not vary periodically in time. However, the two dimensional approximation does have validity under certain circumstances. Moreover, "realistic" flows are obtained from the solution of a set of partial differential equations derived from the physical situation under consideration. Typically, these partial differential equations are strongly nonlinear and can only be "solved" with a computer. This gives rise to a velocity field defined as a data set over a finite time interval, or a finite time dynamical system. Early work on transport in geophysical flows from the point of dynamical systems having aperiodic time dependence and/or defined as a finite time data set can be found in Duan and Wiggins (1996), Miller et al. (1997), Duan and Wiggins (1997), Malhotra and Wiggins (1998), Haller and Poje (1998), Rogerson et al. (1999), and Coulliette and Wiggins (2001). Reviews that describe how these issues in dynamical systems theory arise from the point of view of transport in geophysical flows are Jones and Winkler (2002), Wiggins (2005), Mancho et al. (2006), and Samelson and Wiggins (2006).

This paper is concerned with the extension of the dynamical systems approach to transport in two-dimensional, incompressible flows having more general time dependence than periodic, and their dynamics over a finite time interval. Our focus is on "elliptic behaviour" and invariant tori. However, first we review some of the work on finite time, aperiodically time-dependent dynamical systems. Some of the more recent work in this area has been motivated by the issues raised by some of the work related to transport in geophysical flows noted above. However, there has been a great deal of work in the mathematics community and the control theory community that is relevant that has not been properly recognized. In Sect. 1.1 we describe issues and work on nonautonomous dynamical systems and in Sect. 1.2 we describe issues and work on finite time dynamical systems. 


\subsection{Nonautonomous dynamical systems}

As applied dynamical systems enjoyed an explosion of popularity, starting from the late 1970s and continuing through today, "dynamics" - the study of how the state of a system evolves in time - is typically described by iteration of maps (discrete time) and flows (continuous time). Flows are the group of one-parameter families of transformations of the state space (where the parameter is "time") that are obtained as the solutions of autonomous differential equations (here, we will simplify our discussion by assuming that the solutions of differential equations exist for all positive and negative time). Maps arise naturally from time-periodic differential equations through the so-called "Poincare map" construction. The success of the geometric approach of dynamical systems theory in applications encouraged efforts to extend the ideas to more complex settings, and time-dependent differential equations whose time dependence is more general than periodic are a natural extension to consider. In this case the Poincaré map construction was no longer possible since this relied on the time periodicity of the differential equation. Moreover, the solutions of nonautonomous differential equations do not define flows in the usual sense of the definition ${ }^{1}$. Therefore, the basic consideration that one must begin with is "how do you describe the dynamics arising from a nonautonomous differential equation"?

In some sense, this problem was solved in the 1960s. Dafermos developed the notion of a process and generalized the LaSalle Invariance Principle to this setting (Dafermos (1971)). Miller (1965) and Sell (1967a, b, 1971) developed the notion of skew product flows and their associated cocycle property. These ideas are further described from a pedagogical point of view in the recent review article by Balibrea et al. (2010). With descriptions of "time evolution" appropriate to nonautonomous differential equations at hand, the building blocks of a geometrical theory can be developed. This was begun in the works of Dafermos, Miller, and Sell cited above, but there are other important results from this era that do not appear to be well known. Possibly one reason for this is that the particular nature of the time dependence in the ordinary differential equation community was not so important for many lines of investigation. For example, in the classic ordinary differential equation textbook of Coddington and Levinson (1955) it is easy to see that the proof of the stable and unstable manifold theorem for a hyperbolic trajectory does not use any particular form of time dependence (only that the relevant functions are appropriately bounded in time, and that the existence and uniqueness of solutions holds). A stable and unstable manifold theorem for hyperbolic processes is proven by Irwin (1973) and de Blasi and Schinas (1973), and the more recent textbook by Katok and

\footnotetext{
${ }^{1}$ Specific examples that illustrate the fact that the solutions of nonautonomous equations do not form flows can be found in Balibrea et al. (2010).
}

Hasselblatt (1995) develops the framework of dynamics generated by iteration of sequences of maps, which is a possible framework for nonautonomous dynamics. Fenichel (1991) also proves a stable and unstable manifold theorem for hyperbolic trajectories in discrete time aperiodic systems.

By now the framework for the geometrical analysis of nonautonomous dynamical systems is well under development. Notions of attractivity, stability, and asymptotic behaviour have been developed in Kloeden and Schmalfuss (1997), Langa et al. (2002), Meyer and Zhang (1996), and Sell (1967b, 1971). Shadowing lemmas have been developed in Chow et al. (1989) and Meyer and Zhang (1996). Chaos is discussed and analysed in Lerman and Silnikov (1992), Meyer and Sell (1989), Scheurle (1986), Stoffer (1988a, b), Wiggins (1999), and Lu and Wang (2010, 2011). Various aspects of bifurcation theory are developed in Langa et al. (2002), Poetzsche (2011a, b, 2010b), and Rasmussen (2006). A version of normal form theory is developed in Siegmund (2002). Recent work on general discrete nonautonomous systems is described in Kloeden and Poetzsche (2011) and Poetzsche (2010a).

\subsection{Finite time dynamical systems}

As we mentioned earlier, efforts to use the dynamical systems point of view to analyse transport and mixing in geophysical flows have motivated the study of time-dependent velocity fields that are only defined over a finite time interval, or finite time dynamical systems. Initially, one might think that such a notion is completely at odds with the "dynamical systems point of view", since it is often stated that dynamical systems theory is concerned with the "long time behavior" of a system. Indeed, notions such as "stability" and "attraction" describe aspects of the behaviour of trajectories as time approaches infinity. Mathematical proofs of characteristics of collections of trajectories such as "invariance" and "chaos" typically require an appropriate type of control over these collections of trajectories as time approaches infinity. Nevertheless, computer simulations of a wide variety of dynamical systems (necessarily for a finite simulation time) indicate that these infinite time notions provide both a language and structure to describe the results, and this provides some hope that there is a reasonable chance of success for developing analogous "dynamical systems ideas" for nonautonomous dynamical systems that are only defined for a finite time.

There has been a great deal of activity in recent years in developing a "dynamical systems framework" for finite time dynamical systems. However, it should be noted that similar to the situation described above, the differential equations and control theory communities addressed a number of essential issues in this area many years earlier (and it continues to be a topic of interest in control theory). A recent review paper of Dorato (2006) gives an overview of and historical perspective on work on "finite time stability". The paper of Weiss and Infante (1965) also provides a very insightful 
and rigorous discussion on finite time stability. More recently, Duc and Siegmund (2008) developed basic building blocks (i.e. hyperbolic trajectories and their stable and unstable manifolds) for two-dimensional, time-dependent Hamiltonian systems defined only for a finite time interval. Further work along these lines can be found in Berger et al. (2008). The original definition of "hyperbolicity" is intimately connected to a type of infinite time average along trajectories. Restricting such averages to finite time can be problematic. This topic is treated in Duc and Siegmund (2011) and Berger (2011). Another approach to the notion of hyperbolicity for finite times would be through a proper generalization of the idea of the spectrum associated with the linearization about a finite time trajectory. This is discussed in Berger et al. (2009) and Doan et al. (2011).

With an approach for defining and computing hyperbolic trajectories for finite dimensional vector fields in hand, it is natural to consider the computation of the stable and unstable manifolds of the finite time hyperbolic trajectories. This issue had been considered in Haller (2000), Mancho et al. (2004), Mancho et al. (2006), and Branicki and Wiggins (2010). However, it is important to point out a characteristic associated with finite time hyperbolic phenomena nonuniqueness. In general, all methods used to prove the existence of unique invariant manifolds require the use of a type of iterative or recursive technique with a passage to a limit, and a unique invariant manifold is obtained in this limit. Essentially, passage to the limit means taking time to plus or minus infinity (depending on whether or not one is computing unstable or stable manifolds, respectively). Nevertheless, with respect to the notion of barriers to transport, this is not an issue, since the manifolds are constructed (numerically) with trajectories (and therefore uniqueness of solutions implies that trajectories cannot cross manifolds constructed in this way). The nonuniqueness effectively means that the region where the one-dimensional manifolds (in two space dimensions) are numerically constructed has a certain thickness (see estimate in Haller, 2000) which would go to zero if it were possible to allow time to approach infinity.

There is an important point to be made here which will serve to introduce that aspect of aperiodically timedependent dynamics over finite time intervals that we will be considering in this paper. We emphasize again that we will be considering two-dimensional, time-dependent Hamiltonian systems, i.e. incompressible, two-dimensional velocity fields. Broadly speaking, the stability properties of trajectories and invariant manifolds of Hamiltonian systems are either hyperbolic or elliptic in nature. ${ }^{2}$ Very generally, hyperbolic properties of trajectories, or invariant manifolds, are somewhat independent of any "special structure" of the dy-

\footnotetext{
${ }^{2}$ Of course, this is a bit too simplistic, but it is accurate for our needs. The "boundary" between hyperbolic and elliptic is where bifurcation occurs and requires careful consideration, and "partial hyperbolicity" is also of much current interest (Pesin, 2004).
}

namical system, such as Hamiltonian structure. For example, theorems concerning hyperbolic trajectories and their stable and unstable manifolds are generally equally valid in both Hamiltonian and non-Hamiltonian systems. Stability results for "elliptic dynamics", on the other hand, generally rely crucially on the special structure of the dynamical system (e.g. Hamiltonian, reversible) as well as the coordinates in which the Hamiltonian system is expressed (e.g. action-angle coordinates), with the latter being important for specific analytical methods, such as Fourier analysis. One way of understanding this difference is that "hyperbolic phenomena" are generally stable under perturbation of the dynamical system, while "elliptic phenomena" are not. This would seem to indicate that the fate of "elliptic objects" under perturbation requires a more careful analysis of the effect of the perturbation, and this tends to be the case.

The KAM and Nekhoroshev theorems are major results in Hamiltonian dynamics that are concerned with the behaviour of "elliptic objects", i.e. invariant tori, under perturbation. A standard (and the original) setting for these theorems in (canonical) Hamiltonian systems is that of the (Hamiltonian) perturbation of an integrable system expressed in action-angle variables, i.e. the unperturbed Hamiltonian is expressed entirely as a function of the action variables. This is the setting relevant to us, but more general settings can be found in Broer et al. (1996).

The foundations of the KAM theorem were laid in the 1950s and 1960s (Kolmogorov, 1954; Arnold, 1963; Moser, 1962). Succinct overviews of the essential points of KAM theory can be found in Chierchia and Mather (2010), Pöschel (2001), and Sevryuk (2003). It is probably fair to say that KAM theory became known throughout the worldwide dynamics community from the late 1970s onward. However, the Nekhoroshev theorem came much later (Nekhoroshev (1977)), despite the fact that the phenomenon of "stability over exponentially long time scales" was considered earlier than the KAM theorem in Littlewood $(1959 \mathrm{~b}$, a) and Moser (1955). The Nekoroshev theorem was promoted in the west by the Italian schools associated with Benettin, Gallavotti and Giorgilli. A very accessible proof of the theorem was given in Benettin and Gallavotti (1986), and the website of Prof. Antonio Giorgilli (http://www.mat.unimi.it/ users/antonio/) has a wealth of information on both the KAM and Nekhoroshev theorems, as well as a collection of instructive pedagogical articles and applications to fundamental problems in physics.

We now describe the aspects of the KAM and Nekhoroshev theorems that set the context for the purposes of this paper. For more general settings and conditions of applicability, we refer to the references given above. 


\section{The Kolmogorov-Arnold-Moser (KAM) theorem and Nekhoroshev's theorem}

We begin by considering the more familiar autonomous cases for the KAM and Nekhoroshev theorems. The nonautonomous cases, relevant to our work, are discussed in Sects. 2.4 and 3 .

We consider a Hamiltonian of the following form:

$$
H(I, \theta)=H_{0}(I)+\epsilon H_{1}(I, \theta), \quad(I, \theta) \in B \times \mathbb{T}^{n},
$$

where $B \subset \mathbb{R}^{n}$ is the ball of radius $R$ in $\mathbb{R}^{n}, H_{0}(I)$ is referred to as the unperturbed part of the Hamiltonian and $H_{1}(I, \theta)$ is referred to as the perturbation. The Hamiltonian function needs to be "sufficiently differentiable" on a "wellcontrolled domain", and we will address this issue more precisely when we consider aperiodic time-dependent Hamiltonians in Sect. 3. The coordinates $(I, \theta) \in B \times \mathbb{T}^{n}$ play a very important role. These are the so-called action-angle variables that arise from the structure of the unperturbed, integrable system (Arnold, 1978), and we will have more to say about their role shortly.

The unperturbed Hamiltonian vector field is given by:

$\dot{I}=-\frac{\partial H_{0}}{\partial \theta}(I)=0$.

$\dot{\theta}=\frac{\partial H_{0}}{\partial I}(I)$

and the trajectories of this vector field are given by:

$I(t)=I_{0}=$ constant

$\theta(t)=\frac{\partial H_{0}}{\partial I}\left(I_{0}\right) t+\theta_{0}$.

Clearly, the $n$-dimensional action variable $I=I_{0}$ is constant in time, and the $n$-dimensional angle variables increase linearly in time at a rate defined by the frequency vector $\frac{\partial H_{0}}{\partial I}\left(I_{0}\right)$. In this way $I=I_{0}$ defines an $n$-dimensional invariant torus and the trajectories on the torus are quasiperiodic, having $n$ frequencies.

\subsection{The KAM theorem and sufficient conditions for its application}

The KAM theorem is concerned with the preservation of invariant $n$ tori upon perturbation by the term $H_{1}(I, \theta)$. First, we consider the preservation of a given torus $I=I_{0}$. This torus will "persist" for the perturbed system with the same frequencies $\frac{\partial H_{0}}{\partial I}\left(I_{0}\right)$ provided the unperturbed Hamiltonian satisfies a nondegeneracy condition, the vector of $n$ frequencies is "strongly nonresonant" and the perturbation is sufficiently small. We discuss the sufficient conditions for its application below.

Action-Angle variables. We assume that the unperturbed system is integrable in a way that action-angle variables exist, i.e. there are $n$ integrals that are independent and in involution (these terms and conditions are defined in Arnold,
1978). The full Hamiltonian, i.e. the unperturbed part and the perturbed part, is then expressed in terms of the actionangle variables of the unperturbed, integrable Hamiltonian (cf. Eq. 3). This is important because a number of the analytical methods used in the proofs of the KAM and Nekhoroshev theorems use characteristics of the action-angle variables.

Dealing with Resonances. The KAM theorem is concerned with the preservation under perturbation of certain nonresonant tori on the unperturbed system. $\frac{\partial H_{0}}{\partial I}\left(I_{0}\right)$ is the vector of frequencies associated with the $n$ torus $I=I_{0}$. The standard nonresonant condition for the torus $I=I_{0}$ that the frequencies must satisfy is:

$$
\begin{aligned}
& \left|k \cdot \frac{\partial H_{0}}{\partial I}\left(I_{0}\right)\right|>\gamma\|k\|^{\tau}, \gamma>0, \tau>n-1 \text { for all } \\
& \text { nonzero integer vectors } k=\left(k_{1}, \ldots, k_{n}\right) \in \mathbb{Z}^{n}-\{0\},
\end{aligned}
$$

where $\|k\| \equiv \sum_{i=1}^{n}\left|k_{i}\right|$. It is a standard result that "almost all" (in the sense of Lebesgue measure) frequencies satisfy such a condition (Broer et al., 1996; Chierchia and Mather, 2010).

Nondegeneracy. Nonresonance is a condition on the first derivative of the unperturbed Hamiltonian. Nondegeneracy is a condition on the second derivative. A standard nondegeneracy condition is the following:

$\operatorname{det}\left(\frac{\partial^{2} H_{0}}{\partial I^{2}}\left(I_{0}\right)\right) \neq 0$

If the Hamiltonian depends explicitly on time, a different nondegeneracy condition is used, the so-called isoenergetic nondegeneneracy condition. This is the standard nondegeneracy condition, but restricted to a level set of the Hamiltonian. For details see Broer et al. (1996) and Chierchia and Mather (2010).

\subsection{The Nekhoroshev theorem and sufficient conditions for its application}

Now we turn our attention to Nekhoroshev's theorem (Nekhoroshev, 1977). First we state the theorem in the form of a "model statement" (Lochak, 1993), describe what this means, and then give some background and history. Our statement applies to the Hamiltonian Eq. (3) (and the Hamiltonian must be analytic on an appropriate domain; we will comment more on this later).

For an initial condition $I(0) \equiv I_{0} \in B$ we have:

$\left\|I(t)-I_{0}\right\| \leq c_{1} \varepsilon^{b}$ for $|t| \leq \exp \left(c_{2} / \varepsilon^{a}\right)$

for $\varepsilon \leq \varepsilon_{0}$. Here $\varepsilon$ is a parameter that is estimated in the proof of Nekhoroshev's theorem in terms of the defining parameters of the Hamiltonian (to be discussed later on); $\varepsilon_{0}$ is a "threshold" value for $\varepsilon$. The parameters $\varepsilon_{0}, c_{1}$, and $c_{2}$ are also estimated in terms of the defining parameters of the Hamiltonian, and the "stability exponents" $a$ and $b$ are estimated as functions of $n$. 
Just as we did for the KAM theorem above, we summarize the sufficient conditions for the applicability of Nekhoroshev's theorem below.

Action-Angle variables. As for the KAM theorem, we assume that the unperturbed system is integrable in a way that action-angle variables exist, exactly as we described for the KAM theorem.

Dealing with Resonances. The Nekhoroshev theorem does not focus on specific values of $I$ corresponding to nonresonant tori. Rather, it provides an estimate of evolution in time of any initial action variable over an exponentially long time. The proof of the Nekhoroshev theorem is divided into two parts - an analytic part and a geometric part. The analytic part derives a normal form that is valid in a particular type of nonresonance region. Estimates of the evolution of the action variables can then be obtained for that particular region. The geometric part is the creative element provided by Nekhoroshev. He developed a method that enabled him to show that the entire phase space could be covered by domains in such a way that the normal forms appropriate to these domains, and the associated estimates of the evolution of the action variables, applied to the entire phase space. This construction requires the nondegeneracy condition that we next describe. The geometric argument was improved in Pöschel (1993). A pedagogical discussion of the geometric argument is given in Giorgilli (2002).

Nondegeneracy. The unperturbed Hamiltonian must satisfy a nondegeneracy condition, i.e. a condition on the second derivative of the unperturbed Hamiltonian. However, it is different from the nondegenderacy condition of the KAM theorem. A standard nondegeneracy condition is that the unperturbed Hamiltonian must satisfy a convexity condition of the following form. In particular,

$$
\begin{aligned}
& \left\|\frac{\partial^{2} H_{0}}{\partial I^{2}}(I) v\right\| \leq M\|v\|, \quad\left|\frac{\partial^{2} H_{0}}{\partial I^{2}}(I) v \cdot v\right| \geq m\|v\|^{2} \\
& \text { for all } v \in \mathbb{R}^{n}, m \leq M .
\end{aligned}
$$

Similar to the KAM case, a different nondegeneracy condition may be applied when the perturbation depends explicitly on time. In this case it is assumed that the unperturbed Hamiltonian is quasiconvex, i.e. it is convex on a fixed level set of the Hamiltonian. For details see Broer et al. (1996). In his original proof Nekhoroshev used a weaker nondegeneracy condition referred to as "steepness"; see Nekhoroshev (1977).

\section{The idea behind "exponential stability" estimates}

"Exponential stability" estimates are not obtained from a "straightforward" application of perturbation theory. Here we give a brief, non-rigorous, discussion of how exponential stability estimates can be obtained from a perturbation expansion. "Non-rigorous" means we do not provide proper estimates of domains and sizes of the remainder in the perturba- tion expansion. These details are at the heart of the Nekhoroshev theorem. Rather, we show how an exponentially small remainder of a perturbation series can be obtained if a perturbation series of a particular type is, somehow, obtained. We discuss the autonomous case since the argument is simpler, and it suffices to convey the main ideas behind exponentially small stability estimates. Our discussion follows from Giorgilli (1995).

We consider the following "near integrable" autonomous Hamiltonian:

$H(I, \theta)=H_{0}(I)+\varepsilon \tilde{H}(I, \theta), \quad I \in B \in \mathbb{R}^{n}, \theta \in \mathbb{T}^{n}$,

where $B$ is the open ball centered at the origin of radius $R$. The associated Hamiltonian vector field is given by:

$\dot{\theta}=\frac{\partial H}{\partial I}(I, \theta)$,

$\dot{I}=-\frac{\partial H}{\partial \theta}(I, \theta)$,

and we are interested in the time evolution of the $I$ variables.

We suppose that " $r$ steps" of canonical transformation theory have been performed, which transform Eq. (10) into the normal form:

$$
\begin{aligned}
H^{\prime}\left(I^{\prime}, \theta^{\prime}\right) & =H_{0}\left(I^{\prime}\right)+\varepsilon H_{1}\left(I^{\prime}\right)+\cdots \\
& +\varepsilon^{r} H_{r}\left(I^{\prime}\right)+\varepsilon^{r+1} R\left(I^{\prime}, \theta^{\prime}\right) .
\end{aligned}
$$

The nature of the domain and the properties of the canonical transformations on this domain that bring Eq. (10) into the form of Eq. (11) are important ingredients in Nekhoroshev's theorem. However, they are not important for the point that we wish to discuss here. Rather, given a normal form of the form Eq. (12) we will describe, roughly, how one obtains an exponential estimate (and, in course, what exactly this italicized phrase means) ${ }^{3}$. Hamilton's equations for the Hamiltonian Eq. (12) are given by:

$\dot{\theta}^{\prime}=\frac{\partial H^{\prime}}{\partial I^{\prime}}\left(I^{\prime}, \theta^{\prime}\right)=\frac{\partial H_{0}}{\partial I^{\prime}}\left(I^{\prime}\right)+\mathcal{O}(\varepsilon)$,
$\dot{I}^{\prime}=-\frac{\partial H^{\prime}}{\partial \theta^{\prime}}\left(I^{\prime}, \theta^{\prime}\right)=-\varepsilon^{r+1} \frac{\partial R}{\partial \theta^{\prime}}\left(I^{\prime}, \theta^{\prime}\right)$.

We are interested in the time evolution of the action variables, which are given by:

$I^{\prime}(t)-I^{\prime}(0)=-\varepsilon^{r+1} \int_{0}^{t} \frac{\partial R}{\partial \theta^{\prime}}\left(I^{\prime}(\tau), \theta^{\prime}(\tau)\right) d \tau$,

\footnotetext{
${ }^{3}$ Of course, the real innovation of Nekhoroshev was showing how the entire action space could be covered with domains on which "appropriate" normal forms could be constructed (i.e. normal forms that were "adapted" to possible resonances on the domains), with associated exponential estimates, and how these estimates could be extended to the entire action space.
} 
and therefore

$$
\left|I^{\prime}(t)-I^{\prime}(0)\right| \leq \varepsilon^{r+1} t\left\|\frac{\partial R}{\partial \theta^{\prime}}\right\|,
$$

where $\|\cdot\|$ denotes an appropriate norm on functions (a discussion of the particular norm is not important for the present discussion). It follows from these estimates that:

$$
\left|I^{\prime}(t)-I^{\prime}(0)\right|=\mathcal{O}(\varepsilon) \quad \text { if } \quad \epsilon^{r+1} t\left\|\frac{\partial R}{\partial \theta^{\prime}}\right\|=\mathcal{O}(\varepsilon),
$$

and, therefore, we will have $\left|I^{\prime}(t)-I^{\prime}(0)\right|=\mathcal{O}(\varepsilon)$ on a time interval $[0, T]$ where

$$
T=\frac{\mathcal{O}(\epsilon)}{\varepsilon^{r+1}\left\|\frac{\partial R}{\partial \theta^{\prime}}\right\|} .
$$

Now if $\left\|\frac{\partial R}{\partial \theta^{\prime}}\right\|$ the result obtained is just the standard perturbation theory estimate after $r$ steps of the normalization process, we have $\left|I^{\prime}(t)-I^{\prime}(0)\right|=\mathcal{O}(\varepsilon)$ on a time interval of length $\mathcal{O}\left(\frac{1}{\varepsilon^{r}}\right)$. The problem with this conclusion is that $\left\|\frac{\partial R}{\partial \theta^{\prime}}\right\|$ is not bounded. In general it grows as some power of $r$ ! (such an estimate is obtained in the course of the proof of Nekhoroshev's theorem). There is also the fact that an arbitrary parameter $r$ should not play a role in the form of a stability result, but this point will also be addressed in the course of our discussion. Therefore, in general we expect $H_{r}$ to have the estimate $\mathcal{O}(r !)$. We will ignore constants since they are not essential for understanding the essence of the manner for obtaining exponential stability results. Roughly, in order for Eq. (12) to be of use, the ratio of the order $r+1$ term to the order $r$ term must be smaller than 1, i.e.

$$
\frac{(r+1) ! \epsilon^{r+1}}{r ! \epsilon^{r}}=(r+1) \varepsilon<1 .
$$

This immediately suggests an "optimal" form for $r$ in terms of $\varepsilon$ :

$r+1 \approx \frac{1}{\varepsilon}$.

Now recall Stirling's formula:

$r ! \approx \sqrt{r} r^{r} e^{-r}$

Since the remainder term is of the order $(r+1) ! \varepsilon^{r+1}$, substituting Eqs. (19) and (20) into this expression will give an expression for the order of the remainder in terms of the "optimal" normalization order as a function of $\varepsilon$ :

$$
\begin{aligned}
(r+1) ! \varepsilon^{r+1} & \approx \sqrt{r+1}(r+1)^{r+1} \varepsilon^{r+1} e^{-(r+1)}, \\
& \approx((r+1) \varepsilon)^{r+1} \sqrt{r+1} e^{-(r+1)}, \\
& \approx 1 \sqrt{\frac{1}{\varepsilon}} e^{-\frac{1}{\varepsilon}}
\end{aligned}
$$

Hence, we see that with this choice of $r$ the remainder term in Eq. (12) is "exponentially small in $\varepsilon$ ", and using this result with Eq. (17) gives the exponential stability estimate. Of course, we avoided many details that must be dealt with in the course of proving the Nekhoroshev theorem. However, this is the essence of the idea, given a normal form of the form of Eq. (12). A great deal of additional work is required to then show that the entire phase space can be covered with regions on which "appropriate normal forms" having exponential stability estimates are valid, and that these estimates can be used to give a "uniform" estimate valid for the entire phase space (this is the "geometric part" of the Nekhoroshev theorem).

\subsection{Verifying that the assumptions of the KAM and Nekhoroshev theorems hold in specific examples}

The conditions for the validity of the KAM theorem and the Nekhoroshev theorem in specific applications appear straightforward. However, this situation is somewhat misleading. Most of the work that verifies the applicability of the KAM and Nekhoroshev theorems for specific models has been carried out in the context of models in celestial mechanics; see, e.g., Celletti and Chierchia (2007) and Giorgilli et al. (2009). A specific model problem where detailed calculations of the applicability of Nekhoroshev's theorem are carried out is described in Lochak and Porzio (1989). The issues with applicability start at the very beginning of the consideration of the application. The KAM and Nekhoroshev theorems are stated, and proven, using the action-angle variables of the unperturbed integrable system. Even if one has a model that can be divided into an integrable part plus "a perturbation", it is, in general, highly nontrivial to construct action-angle coordinates for the unperturbed, integrable part. For this reason there have been essentially no applications of the KAM theorem to fluid transport where the conditions for the applicability of the theorem have been verified for a model under consideration. Similarly for the Nekhoroshev theorem, although that result hardly seems known at all by those considering Lagrangian transport issues in the fluids community.

Nevertheless, the KAM theorem provides a "language" to discuss invariant tori, and their manifestation as flow barriers, even though the applicability of the theorem is generally not verified for specific flows. The reason for this is the nature of the KAM theorem itself, and the conditions for its applicability. In particular, we know that, for the unperturbed (two-dimensional, time-independent and incompressible) flow, in a region of closed streamlines action-angle variables exist theoretically, even if we cannot find analytical expressions for the explicit coordinates (Arnold, 1978). Moreover, the nonresonance and nondegeneracy conditions are generic. Therefore it would be surprising if they did not hold. Nevertheless, this is no substitute for a quantitative study of the limits of applicability of these theorems in specific examples. For promising recent work on the applicability of KAM 


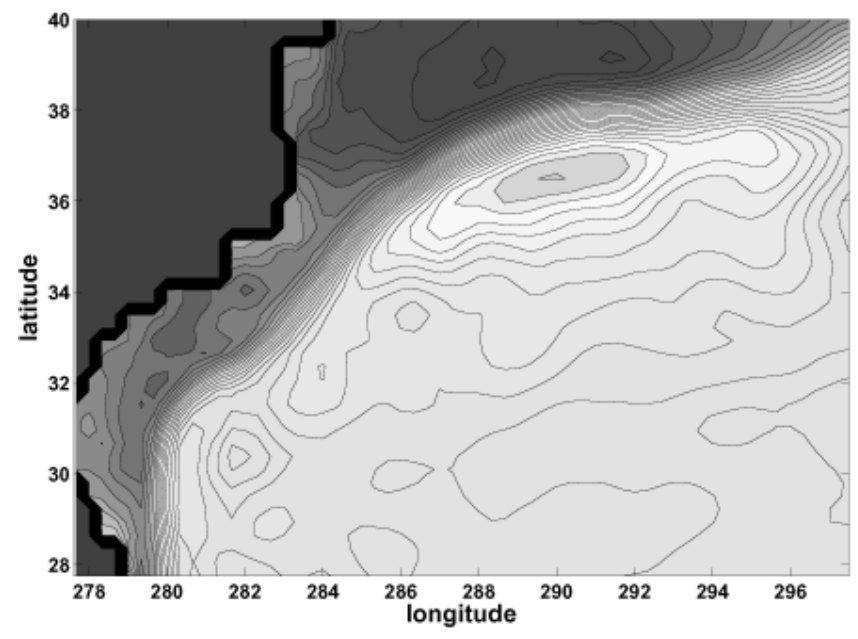

Fig. 1. Average sea-surface height, which is related to streamlines of the velocity field, in the North Atlantic. The "jet" is clearly observable. Image courtesy of Jezabel Curbelo.

theory without action-angle variables see de la Llave et al. (2005).

\subsection{Application of the KAM and Nekhoroshev theorems to fluid transport}

By now it is "common knowledge" that in the application of the KAM theorem to fluid transport the KAM tori act as complete "barriers to transport". This statement requires much more careful consideration and we want to explore its meaning and full implications in terms of our discussion of the KAM and Nekhoroshev theorems discussed above. First, we motivate our discussion by considering a particular "flow feature" that arises in many geophysical flow studies: a jet. The discussion is also very relevant to our discussion in Sect. 2.3.

A two-dimensional (2-D) "meandering jet" is a common flow feature observed on the surface of the ocean. In particular, specific 2-D jets that are visible on the ocean's surface are, for example, the Gulf Stream and the Kuroshio currents. These circulating patterns are very stable and are often described from the perspective of a stationary reference flow plus a (temporal) variability that acts as a not too large aperiodic perturbation of the reference state (note that we do not expect geophysical flows to have time-periodic or quasi periodic variability, even though this has been the subject of investigation for many kinematic jet models). Figure 1 shows the time average of the sea-surface height (SSH) in the North Atlantic. The magnitude of the SSH is locally related to a stream function from which the surface velocity is obtained in the geostrophic approximation. A very similar picture can be obtained for the Kuroshio region.

Consequently, kinematically defined 2-D meandering jet models have received much attention over the years. The specific details of those models are not important for our discussion, only the geometry of the streamlines - and the coordinates. However, some selected relevant references are Bower (1991), Samelson (1992), Duan and Wiggins (1996), and Samelson and Wiggins (2006), and in these references a specific functional form for the flow field of this particular model of a jet can be found. In a frame of reference moving with the phase velocity of the jet, the streamlines appear as in Fig. 2a. In particular, the flow is steady and spatially periodic. It is important to realise that the horizontal and vertical coordinates in Fig. 2a are the physical coordinates describing the streamlines of the jet, i.e. they are not action-angle coordinates. The left and right vertical boundaries of the flow shown in Fig. 2a are identified, i.e. the flow is periodic in the horizontal direction. Consequently, there are five regions of closed trajectories, denoted $R_{1}, \ldots, R_{5}$ in the figure. The jet is the central region, denoted $R_{3}$ (these trajectories are periodic since the flow is spatially periodic). Immediately above and below the jet are regions of "recirculating trajectories", denoted $R_{2}$ and $R_{4}$, and at the very top and bottom are two regions of periodic trajectories, denoted $R_{1}$ and $R_{5}$, that traverse the entire domain and move in the opposite direction as the jet (i.e. trajectories in $R_{3}$ ).

This flow structure describes a steady, incompressible, two-dimensional flow (hence, it is Hamiltonian and integrable) having five regions of qualitatively distinct closed trajectories (we have not considered variability applied to this model - yet). In order to apply the KAM and Nekhoroshev theorems to this flow we must transform the flow to action-angle variables in the regions of closed trajectories. However, the action-angle transformations for the five different regions will generally be different, and action-angle coordinates are not defined on the separatrices that separate the five regions. This is something of a moot point since the transformation to action-angle coordinates, for even one of the regions, has not been carried out for any of the kinematically defined jet models noted above. What is required is that this transformation produces a change of coordinates in which the transformed variables are as follows. The horizontal coordinate (the angle) must be periodic. Additionally, in the new coordinates, the streamlines or contour lines of the Hamiltonian must have a geometry compatible with expression (3). This means that the Hamiltonian must depend, to leading order, on the vertical coordinate (the action) plus a small distortion introduced by the perturbation $H_{1}$. This means that for instance the lines in the transformed region of interest of Fig. 2a, before the addition of the perturbation, should be purely horizontal. Following the expression found in Samelson (1992) for the Hamiltonian displayed in Fig. 2a, the unperturbed term $H_{0}$ depends both on the horizontal and vertical variables, thus it would not be in the appropriate coordinate system required by the Nekhoroshev theorem. We will consider an example in Sect. 4 that is expressed from the beginning in action-angle variables, that has the geometric features of the jet, and therefore allows us to apply the Nekhoroshev theorem with a variety of time dependencies. 


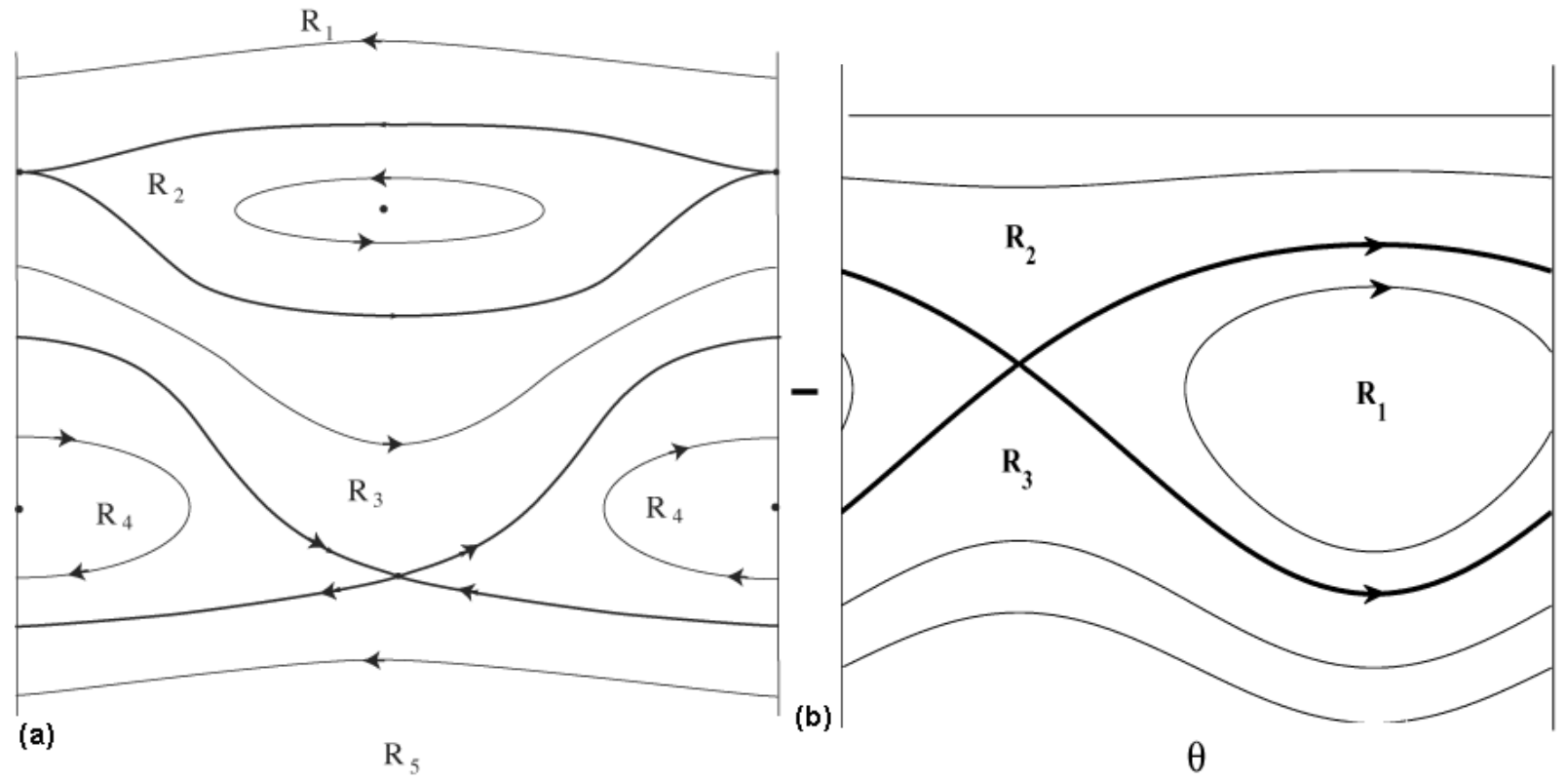

Fig. 2. (a) Streamlines associated with a "jet" (figure from Samelson and Wiggins, 2006) shown in the physical coordinates of the flow; (b) streamlines associated with the "jet" example from Sect. 4 shown in the action-angle variables. The streamlines are shown for $b(t)=0$ and $\varepsilon=0.1$.

Figure $2 b$ shows the streamlines of the particular example that will be the focus of our study in Sect. 4. This example shows patterns similar to that of Fig. 2a, with a recognizable recirculating region $R_{1}$ and two regions $R_{2}$ and $R_{3}$ with periodic trajectories that transverse the entire domain (the example is periodic in the horizontal direction). This flow is different, though, from the kinematic models illustrated in Fig. 2a in that it is obtained from a Hamiltonian expressed in actionangle variables that correspond to the vertical and horizontal axes, respectively. In particular, the example Hamiltonian in Sect. 4 has the form $H(I, \theta)=H_{0}(I)+\varepsilon H_{1}(I, \theta, t)$ so that the Nekhoroshev theorem can be applied immediately. For the streamlines shown in Fig. 2b we have chosen $\varepsilon=0.1$ and $H_{1}(I, \theta, t)=H_{1}(I, \theta)$ by setting $b(t)=0$.

Even if we succeed in expressing the stream function in action-angle coordinates in the regions of interest of the flow, we note that the general Hamiltonian given in Eq. (3) will only have relevance as a stream function of a fluid flow for the case $n=1$. In this case it would describe a steady, twodimensional incompressible flow (in action-angle variables). This is not particularly interesting (from the point of view of mixing, but possibly for transport), since two-dimensional, steady incompressible flows are integrable, and in this case the integral is $H(I, \theta)=H_{0}(I)+H_{1}(I, \theta), \quad(I, \theta) \in B \times \mathbb{T}$, where $B$ is an interval in $\mathbb{R}$. Therefore in order for there to be "interesting" mixing and transport in two dimensions, the flows must be time dependent, from which it follows that if the KAM and Nekhoroshev theorems are to play an important role, then the corresponding Hamiltonian must be time dependent:

$H(I, \theta, t)=H_{0}(I)+\varepsilon H_{1}(I, \theta, t), \quad(I, \theta) \in B \times \mathbb{T}$.

The particular type of time dependence that has been studied in some detail is that of quasiperiodic time dependence. This means that the time-dependent perturbation can be written in the following form:

$H_{1}(I, \theta, t) \equiv H_{1}\left(I, \theta, \phi_{1}, \ldots, \phi_{m}\right)$,

where $H_{1}\left(I, \theta, \phi_{1}, \ldots, \phi_{m}\right)$ is $2 \pi$ periodic in each $\phi_{k}, k=$ $1, \ldots, m$ (for each $I, \theta$ ) where $\phi_{k}=\omega_{k} t$ for $k=1, \ldots, m$. Of course, the case $m=1$ corresponds to the time-periodic case.

Some early results on periodic and quasiperiodic time dependence of the KAM theorem are discussed in Arnold et al. (1988). More recent results can be found in Broer et al. (1996) and Sevryuk (2007). A detailed discussion, and theorem, for quasiperiodically time-dependent Hamiltonian systems with Hamiltonians of the form of Eq. (23) can be found in Jorba and Simo (1996). Concerning the nature of the tori that persist under the perturbation, the situation is best described by a passage from this paper.

"The frequencies of these tori are those of the unperturbed tori plus those of the perturbation. This can be described by saying that the unperturbed tori are "quasi-periodically dancing" to the "rhythm" of the perturbation. The tori whose frequencies are in resonance with those of the perturbation are destroyed." (Jorba and Simo, 1996).

Hence, the surviving invariant tori, for one degree-offreedom, $n=1$, form "complete" barriers to transport in the 
sense that these invariant tori are invariant manifolds (i.e. they consist of trajectories) and therefore trajectories not starting on the invariant tori cannot cross the invariant tori. Moreover, by examining the regions of closed trajectories for the "unperturbed" steady flows shown in Fig. 2 one can see that the particular interpretation of these surviving invariant tori in terms of their influence on transport depends on the geometry of the closed streamlines of the unperturbed flow and their relation to the global geometry of the flow. For an application of similar ideas to two-dimensional, quasiperiodically time-dependent flows see Beron-Vera et al. (2010). At present, there is no analogue of the KAM theorem for perturbations having more general time dependence than quasiperiodic, which is particularly notable for geophysical transport applications since one does not expect typical ocean variability to be either periodic or quasiperiodic. However, the observed similarities among the flow structures shown in Figs. 1 and 2 suggest that the transport properties associated with perturbed invariant tori can be related to important transport questions such as, for example, the existence of crossjet transport of radiative isotopes in the Kuroshio current following the Fukushima accident (Buesseler et al., 2012), or the time of persistence of particles within the jet (this latter problem is directly connected to the results of the Nekhoroshev theorem, as we will discuss later), or similar transport issues.

Next, we turn our attention to the Nekhoroshev theorem. This theorem has not received as much attention from the point of view of perturbations with general time dependence, with the major exception of the remarkable paper of Giorgilli and Zehnder (1992).

\section{"Nearly invariant" tori}

Roughly, the estimate in Eq. (8) implies that the action coordinates stay "close" (as measured by some power of a small parameter) to their initial values for a time that is exponentially long (where the exponent is a constant multiplied by the inverse of a (possibly) different power of the same small parameter). The phrases "exponential stability" or "effective stability" are often used. This is a very special type of finite time stability, as eloquently described by Littlewood (Littlewood, 1959b): “... while not eternity, this is a considerable slice of it."

In this situation the term "nearly invariant tori" is used in the literature (see, e.g., Delshams and Gutierrez, 1996), and this notion is particularly relevant for the notion of "invariant tori" finite time dynamics.

The issue of the existence of invariant tori poses related issues for finite time Hamiltonian vector fields with respect to nonuniqueness and the associated inability to locate precise invariant manifolds as discussed in Sect. 1.2. Invariant tori are barriers to transport - they are invariant manifolds and, therefore, trajectories cannot cross invariant tori. How- ever, they are infinite time objects in the sense that their existence is proved by an iterative or recursive process that requires a passage to a limit. Moreover, there is no existing version of a KAM theorem for general aperiodically timedependent Hamiltonian systems (other than for quasiperiodic time dependence). However, the Nekhoroshev theorem may be viewed as a type of finite time KAM theorem in the sense that an invariant torus is identified in the unperturbed system and a thickened region is constructed around that invariant torus in which trajectories starting in that region will remain for an exponentially long time. As we have noted, this is similar in spirit to the issue of nonuniqueness for the stable and unstable manifolds of finite time hyperbolic trajectories, and it is probably as good as one might expect for time-dependent vector fields defined on a finite time interval.

Of course, this raises the issue of how useful this is for applications, since one has not identified an exact barrier to transport that is valid for all time. However, practically, this may not be the essential important element. Rather, identifying regions of the flow where trajectories remain for very long times may be more practical since one can only ever observe flow for a finite time.

\section{The Nekhoroshev theorem for aperiodic time dependence}

Giorgilli and Zehnder (1992) considered time-dependent Hamiltonian systems of the following form:

$$
H(\theta, I, t)=\frac{|I|^{2}}{2}+V(\theta, t), \quad(\theta, I, t) \in \mathbb{T}^{n} \times \mathbb{R}^{n} \times \mathbb{R} .
$$

This Hamiltonian would appear to have little relevance to the fluid transport settings described earlier, since it would be unusual for a stream function to have the form of "kinetic plus potential energy". Moreover, there is no small parameter in Eq. (24) that would give it the form of the problem of the perturbation of an integrable system. However, a closer examination of Giorgilli and Zehnder (1992) reveals that the techniques used in the paper are much more general than the stated results. The main goal in Giorgilli and Zehnder (1992) was to show that the action variables of Eq. (24) remain bounded over exponentially long time intervals. Casting this problem in the "Nekhoroshev setting" requires $|I|$ to be "large", which will make $V(\theta, t)$ a "small" perturbation of $\frac{|I|^{2}}{2}$ (and this is why no small parameter appears in the statement of this problem). However, Giorgilli and Zehnder (1992) showed that by rescaling the action variables and time by a small parameter, Eq. (24) could be transformed to the form of a "slow time varying" perturbation of an integrable system in the "standard sense".

There is still the issue of the special form of the Hamiltonian in Eq. (24). Recall from the earlier discussion that the proof of the Nekhoroshev theorem is in two parts - an analytic part and a geometrical part. The analytic part uses 
standard canonical perturbation theory to derive resonant normal forms on certain regions of phase space. The geometric part shows that the regions on which the normal forms are valid cover the entire phase space. In this way the evolution of trajectories on all of the phase space can be estimated from the dynamics of the normal forms.

It is easy to see that the analytical part in Giorgilli and Zehnder (1992) is very general and does not depend on the special form of the Hamiltonian Eq. (24). The proof of the geometric part given in Giorgilli and Zehnder (1992) is greatly simplified with the special form of Eq. (24). However, this is probably of no consequence for our needs for fluid transport, since in that case we only require $n=1$. Nevertheless, recently Bounemoura (2013) and Fortunati and Wiggins (2013) have re-visited the work of Giorgilli and Zehnder (1992) and provided a formulation of the Giorgilli and Zehnder (1992) result for a standard perturbation of an integrable Hamiltonian system with arbitrary, slowly varying, time dependence.

It is worth noting the issue of "slow time dependence". The results of Giorgilli and Zehnder (1992), Bounemoura (2013) and Fortunati and Wiggins (2013) all require slow time dependence ("slow" in the sense of the time dependence of the Hamiltonian where the explicit time variable is multiplied by some positive power of the perturbation parameter). Giorgilli and Zehnder (1992) and Fortunati and Wiggins (2013) use a different scheme than Bounemoura (2013) to arrive at the necessary normal form. So, at the moment, it appears that slow time dependence is required to achieve a Nekhoroshev result for aperiodically time-dependent systems and that this should be regarded as the most general form for a Hamiltonian with arbitrary time dependence.

We now state the theorem in a form that is adequate for our needs.

We consider a one degree-of-freedom, aperiodically timedependent Hamiltonian of the following form:

$$
\begin{aligned}
& H(\theta, I, t)=H_{0}(I)+\varepsilon \tilde{H}\left(\theta, I, \epsilon^{c} t\right), \\
& (\theta, I, t) \in \mathbb{T} \times \mathcal{D}_{\rho} \times \mathbb{R},
\end{aligned}
$$$$
\text { where } \mathcal{D}_{\rho} \text { is a ball of radius } \rho \text { in } \mathbb{R} \text { and } \frac{1}{2} \leq c \leq 1 \text {, }
$$

with corresponding Hamiltonian vector field:

$$
\begin{aligned}
\dot{\theta} & =\frac{\partial H_{0}}{\partial I}(I)+\varepsilon \frac{\partial \tilde{H}}{\partial I}\left(\theta, I, \varepsilon^{c} t\right), \\
\dot{I} & =-\varepsilon \frac{\partial \tilde{H}}{\partial \theta}\left(\theta, I, \varepsilon^{c} t\right) .
\end{aligned}
$$

It is well known that a time-dependent Hamiltonian can be cast in the form of a time-independent Hamiltonian with an additional degree of freedom. This formulation allows one to treat the problem by an analytic part of the problem by standard canonical perturbation theory, as explained in Giorgilli and Zehnder (1992). We will not be pursuing the proof of the theorem here. However, it is useful to cast the problem in this form in order to understand the role that certain parameters play in the formulation of the result. Towards this end, we re-write the Hamiltonian by redefining a new variable as $\xi=\varepsilon^{c} t$ and introducing a new extra variable $\eta$ in an extra term in the following form:

$H(\theta, I, \xi, \eta)=H_{0}(I)+\varepsilon^{c} \eta+\epsilon \tilde{H}(\theta, I, \xi)$.

The corresponding Hamiltonian vector field is:

$$
\begin{aligned}
\dot{\theta} & =\frac{\partial H_{0}}{\partial I}(I)+\varepsilon \frac{\partial \tilde{H}}{\partial I}(\theta, I, \xi), \\
\dot{I} & =-\varepsilon \frac{\partial \tilde{H}}{\partial \theta}(\theta, I, \xi), \\
\dot{\xi} & =\frac{\partial H}{\partial \eta}(\theta, I, \xi, \eta)=\varepsilon^{c}, \\
\dot{\eta} & =-\varepsilon \frac{\partial \tilde{H}}{\partial \xi}(\theta, I, \xi) .
\end{aligned}
$$

The variables $(I, \theta, \xi, \eta)$ are now extended to the complex plane $\mathbb{C}$. We define:

$\mathcal{G}_{\delta}=\left\{I \in \mathbb{C}|| I-\mathcal{D}_{\rho} \mid<\delta\right\}$,

then the following domain for the Hamiltonian for Eq. (27) is considered:

$\mathcal{G}_{(\delta, \sigma)}=\mathcal{G}_{\delta} \times\{\eta \in \mathbb{C}\} \times\{|\operatorname{Im} \theta|<\sigma\} \times\{|\operatorname{Im} \xi|<\sigma\}$.

Additionally, we have the following assumptions.

Analyticity The Hamiltonian Eq. (27) is analytic on the domain Eq. (29).

Nondegeneracy of the Integrable Part For $n=1$ the nondegeneracy condition on the integrable part is particularly simple:

$M>\left|\frac{\partial^{2} H_{0}}{\partial I^{2}}\right|>m>0, \quad$ for some $M>m>0$,

where $M$ and $m$ are upper and lower bounds, respectively, on the magnitude of the frequency.

We can now state the main result due to Giorgilli and Zehnder (1992) (and refined by Bounemoura, 2013 and Fortunati and Wiggins, 2013).

Theorem 1 Under the assumptions given above, there exists positive constants $\varepsilon_{0}, c_{1}, c_{2}, c_{3}$ that depend on $\delta, \sigma, m, M$ such that if $\varepsilon \leq \epsilon_{0}$ such that for all solutions $(\theta(t), I(t))$ of Eq. (26) if $I(0) \in \mathcal{D}_{\frac{\rho}{2}}$ then

$|I(t)-I(0)| \leq c_{1} \varepsilon^{\frac{1}{2}}$,

for all

$$
|t| \leq c_{2} \exp \left(c_{3} \varepsilon^{-\frac{1}{2}}\right) .
$$


We make several comments regarding this theorem.

- It is important to understand what is meant by almost invariant tori in the case where the time dependence is not periodic, since by invariant torus typically it is understood that the motion is quasiperiodic. Recall our discussion of almost invariant tori at the end of Sect. 2.4. An almost invariant torus was identified in the unperturbed system and the Nekhoroshev theorem was used to define a thickened region around that invariant torus in which trajectories starting in that region remained for an exponentially long time. Since the torus is identified in the unperturbed problem, this definition still holds when the perturbation is not periodic in time.

- The issue of the choice for the constants $c_{1}, c_{2}, c_{3}$ is important to note. They are of order 1 and do not depend on the perturbation parameter. Functional forms for them are derived during the course of the proof of the theorem, but general choices are made for various parameters that define the constants and inequalities that arise at different steps in the proof in order to provide a simple and convenient proof of the theorem. Therefore in the proof of the general theorem no effort is made to choose the constants in such a way that is optimal for a specific problem. Moreover, the parameters that go into each constant need to be computed explicitly for each specific example, and for some of the parameters it is not entirely clear how to compute such parameters for a specific example in an optimal manner (e.g. analyticity parameters and ultraviolet cutoff parameters for truncating Fourier series in the normal form). This is why the computations of all of the constants involved in a specific example for application of the KAM theorem (e.g. Celletti and Chierchia, 2007) or the Nekhoroshev theorem (e.g. Lochak and Porzio, 1989) amount to a substantial research project in their own right. It is worth mentioning that it is only in the last 2 years that the nondegeneracy condition for the KAM theorem in the context of the general $n$ body problem of celestial mechanics has been verified (the work of Chierchia and Pinzari, 2011). Nevertheless, even though its rigorous applicability to the $n$ body problem was not established, the KAM theorem provided a valuable theoretical framework for thinking about the problem. We believe that the Nekhoroshev theorem will serve a similar role for finite dimensional fluid transport problems, and this may serve to motivate work on verifying the conditions for the KAM and Nekhoroshev theorems for specific examples that are more directly related to fluid mechanical equations of motion.
For our example in Sect. 4 we take the constants in Nekhoroshev's theorem to be $c_{1}=c_{2}=c_{3}=1$. This choice is arbitrary. They are the simplest order 1 constants. Nevertheless, one can see directly from Eqs. (31) and (32) that confinement of trajectories over exponentially long timescales still holds for general order one choices of constants $c_{1}, c_{2}, c_{3}$ if we take $\varepsilon$ sufficiently small. Indeed, for hyperbolic perturbation methods (such as Melnikov's method for determining the existence of transverse intersections of the stable and unstable manifolds) soft analysis is all that is required (i.e. first-order regular perturbation theory, standard implicit function theorems) and, as a result, specific constants are not computed as the results hold for $\varepsilon$ sufficiently small. For the KAM/Nekhoroshev theorems one requires perturbation results to all orders and in this case the constants must be estimated at each step, and it is natural to include them in the statement of the theorem (although that may make the theorem extremely difficult to penetrate for a non-specialist). Still, the results hold for $\varepsilon$ sufficiently small, but knowing the specific constants enables one to compute how small $\varepsilon$ must be.

- The Nekhoroshev estimates hold regardless of whether the unperturbed torus is resonant or not. This has been investigated in detail for the case of $n$ degree of freedom autonomous Hamiltonian systems, and the results are surprising and somewhat counterintuitive. Briefly, the most resonant regions are the most stable in the sense of Nekhoroshev estimates and the least stable regions in the sense of Nekhoroshev estimates are the least resonant regions, e.g. the KAM tori. This phenomena is described in detail in Benettin and Gallavotti (1986) and Lochak (1992).

- The Nekhoroshev estimates do not say anything about the speed at which trajectories move away from an unperturbed invariant torus. For example, it is not ruled out that a trajectory could rather quickly move to the maximum stability radius and then move very little for the rest of the stability time. Whether or not this happens needs to be considered in the context of specific examples.

\section{An example}

Now we consider an example that "gives us an idea" of the usefulness of Theorem 3. We say "gives us an idea" because we have not computed the threshold value for the perturbations $\left(\varepsilon_{0}\right)$ or the constants $c_{1}, c_{2}, c_{3}$ for this example. Computing these constants would require careful consideration of the proof of the theorem in the context of this particular example. However, for our purposes it is sufficient to know that these are $\mathcal{O}(1)$ constants. With this in mind, the example will 
illustrate some interesting features of Nekhoroshev's result in the context of aperiodic time dependence. This theorem appears to have been unnoticed by the community dealing with transport in finite time velocity fields, thus the example will allow us to study related issues in a setting where the time dependence can be specified. It is our hope that this will inspire further work on the many issues related to finite time transport associated with elliptic phenomena.

We consider a Hamiltonian of the following form:

$$
\begin{aligned}
& H(I, \theta, t)=\frac{I^{2}}{2}+\varepsilon(1-I)(1+b(t)) \sin \theta, \\
& (\theta, I) \in \mathbb{T} \times \mathbb{R},
\end{aligned}
$$

where the unperturbed Hamiltonian, $H_{0}$, is $H_{0}=I^{2} / 2$. Note that, at this point, the time dependence of $b(t)$ is completely arbitrary and can be used to study different aspects of time dependence. For example, $b(t)$ can be chosen to be periodic, quasiperiodic, aperiodic, or to exist only for a finite time. The Hamiltonian vector field corresponding to Eq. (33) is given by:

$$
\begin{gathered}
\dot{\theta}=\frac{\partial H}{\partial I}=I-\varepsilon(1+b(t)) \sin \theta, \\
\dot{I}=-\frac{\partial H}{\partial \theta}=-\varepsilon(1-I)(1+b(t)) \cos \theta .
\end{gathered}
$$

We describe some of the important features of this example that play a role in our numerical experiments.

An Invariant Torus: $I=1$. From Eq. (34) it is clear that that for $I=1, \dot{I}=0$, which proves that $I=1$ is an invariant torus. It is important to note that this is independent of both $\varepsilon$ and $b(t)$.

The Integrable Case: $\varepsilon=0$. For the case $\varepsilon=0$ the vector field is integrable and is given by:

$$
\begin{gathered}
\dot{\theta}=\frac{\partial H}{\partial I}=I, \\
\dot{I}=-\frac{\partial H}{\partial \theta}=0 .
\end{gathered}
$$

This system is clearly integrable. Each value of $I$ corresponds to an invariant circle, and the value of the frequency on the invariant circle is also given by $I$ (hence $I=1$ has frequency one). Note that the invariant circle corresponding to $I=0$ is resonant since its frequency is zero.

We remark that we have avoided the issue described in Sect. 2.4 since the unperturbed system is expressed explicitly in terms of action-angle coordinates, and the perturbed system is also expressed in these coordinates.

The Choice of Time Dependence. For the purpose of numerical experiments, we will consider three types of aperiodic time dependence of the following form:

1. Our first choice is a pulse-like time dependence of the form:

$$
b(t)=\frac{\operatorname{sech}^{2}(\varepsilon t)}{\sqrt{2}}(\sin (\sqrt{3} \varepsilon t)+\cos (\sqrt{2} \varepsilon t)),
$$

Figure $3 \mathrm{a}$ and $\mathrm{b}$ shows the graph of this function for $\varepsilon=0.01$ and $\varepsilon=0.1$ respectively. It has a pulse-like structure which is wider for the smaller $\varepsilon$. In both cases, after a time interval, $b(t)$ becomes essentially zero and the system (34) is essentially autonomous. For this reason the system approaches an integrable system, but it is still a perturbed version of $H_{0}$.

2. A quasiperiodic time dependence:

$$
b(t)=\frac{1}{4 \sqrt{2}}(\sin (\sqrt{3} \varepsilon t)+\cos (\sqrt{2} \varepsilon t)),
$$

Fig. 3c and d shows the graph of this function for $\varepsilon=0.01$ and $\varepsilon=0.1$. It is observed that due to the time scaling a larger $\varepsilon$ produces higher oscillation frequencies.

3. An aperiodic time dependence that is obtained from the chaotic time series of a differential equation. A representation of this time series is shown in Fig. 3e. This series is proportional to the second component of a chaotic trajectory that has been obtained from the integration of a periodically forced Duffing equation (we say "proportional" since we normalise the signal so that it has amplitude one). Since our choice is an arbitrary forcing, in this case we do not rescale the time with $\varepsilon$.

The perturbed system (34) possesses kinematically distinct regions similar to those depicted in Fig. $2 \mathrm{~b}$ and denoted by $R_{1}, R_{2}$ or $R_{3}$, representing, respectively, jets and eddies. In order to obtain a visual representation of the Lagrangian structures of the given examples, similar to what is shown in Fig. 2b, but for the time-dependent case, we will use a recently developed approach based on functions called Lagrangian descriptors (see Mendoza and Mancho, 2010, 2012; de la Cámara et al., 2012; Mancho et al., 2013). The Lagrangian descriptor that we use is based on arc length, and is referred to as $M_{1}$ in Mancho et al. (2013) or as $M$ in Mendoza and Mancho $(2010,2012)$ and de la Cámara et al. (2012, 2013). A precise definition and discussion of $M_{1}$ is given in the references. Contours of the Lagrangian descriptors highlight singular features (which are related to the lack of regularity of the function) and these have been shown to be directly related to "phase space structures". Significantly for our situation, the method is directly applicable to the aperiodically and finite time-dependent cases (unlike Poincaré maps). Lagrangian descriptors as reported in Mancho et al. (2013) are based on the integration of a positive and bounded intrinsic property of a trajectory along the trajectory itself during a time interval of length $2 \tau$. Revealing the dynamical features requires the use of a long enough $\tau$ in order to converge to the singular features. 
a)

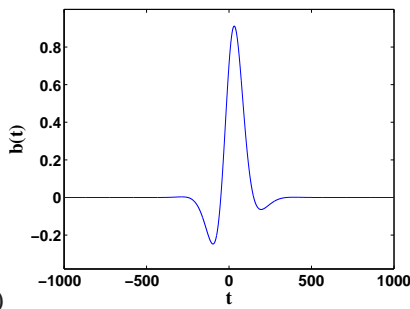

b)

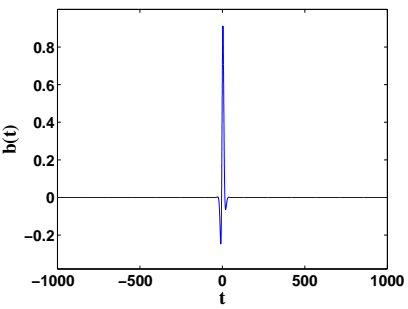

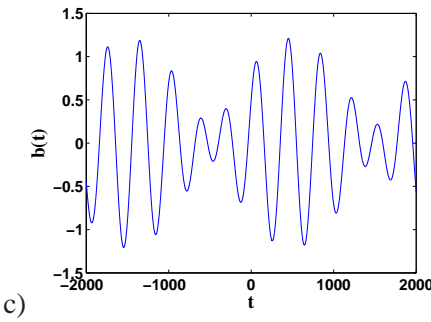

d)
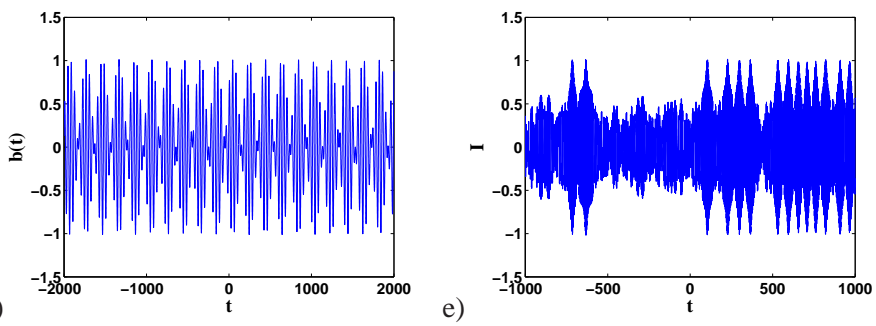

Fig. 3. Representation of different forcing functions $b(t)$ in example (34). (a) Forcing as in Eq. (36) for $\varepsilon=0.01$; (b) forcing as in Eq. (36) for $\varepsilon=0.1$; (c) forcing as in Eq. (37) for $\varepsilon=0.01$; (d) forcing as in Eq. (37) for $\varepsilon=0.1$; (e) chaotic forcing.

\subsection{Numerical experiments}

We will be concerned with the stability properties of three different invariant circles in the unperturbed system:

$I=0$ For the unperturbed system this is a circle of fixed points (i.e. a resonant invariant circle).

$I=\frac{1}{2}$ For the unperturbed system this is an invariant circle with frequency $\frac{1}{2}$.

$I=1$ For the unperturbed system this is an invariant circle with frequency 1 , which also persists as an invariant circle for any value of $\varepsilon$.

We are interested in the stability properties of these three invariant circles. By stability we mean the intuitive idea of "if you start close, you stay close". More precisely, we are interested in the evolution of the action variables of trajectories that "start close" to these invariant circles and whether or not their evolution obeys the Nekhoroshev estimates for "confinement" (Eq. 31) over a given time (Eq. 32).

We consider the following initial conditions for each invariant circle:

$(\theta, I)=(0,0)$

$(\theta, I)=\left(0, \frac{1}{2}\right)$

$(\theta, I)=(0,0.99)$ For this case the action value is "slightly offset" since $I=1$ is invariant for $\varepsilon \neq 0$.

We will consider two values of $\varepsilon$ :

$\varepsilon=0.1,0.01$,

and for each of these values the "confinement time" denoted by $T$ and defined in Eq. (32) is given by:

$T \sim 25, T \sim 22000$, respectively, where we have set $c_{2}=c_{3}=1$. For each of these values of $\varepsilon$ the "confinement distance", denoted by $S$ and defined in Eq. (31), is given by: for these $\epsilon$ values

$S \sim 0.31623, S \sim 0.1$,

respectively, where we have set $c_{1}=1$.

The numerical experiments consist of the following. For each invariant circle, we integrate the initial condition given above and plot the $I$ value of the resulting trajectory as a function of time. This is done for each time dependence and for the two values of $\varepsilon$. Moreover, in order to understand the nature of trajectories we have provided a "snapshot" of the Lagrangian structure through the use of a Lagrangian descriptor. We note that for $\varepsilon=0.1$ we illustrate the trajectory for a time of at least 200 and in some cases up to 400. This is significantly longer than the estimated confinement time of 25. Similarly, for $\varepsilon=0.01$ for all cases we compute the trajectory for a time of 25000 , which is longer than the estimated confinement time of 22000 . For $\varepsilon=0.01$ we will see excellent agreement with the Nekhoroshev estimates for all initial conditions. For $\varepsilon=0.1$ the quality of agreement will vary with the initial condition. This is not unexpected since, a priori, we do not have an estimate of the size of $\varepsilon$, as well as the relevant constants, for each initial condition. The best we can do, in the example under consideration, is to show that "for $\varepsilon$ sufficiently small" the Nekhoroshev estimates hold for trajectories with a particular initial condition. This is typical of how most perturbation theories are applied.

Results for the time dependence (Eq. 36) are shown in Fig. 4. The first column shows the results for $\varepsilon=0.01$ and the second for $\varepsilon=0.1$. It is easily seen that for the case $\varepsilon=0.01$ the Nekhoroshev theorem is satisfied, as the trajectories remain within a distance of $S \sim 0.1$ from the initial condition for at least $T \sim 22000$ time units. 
a)
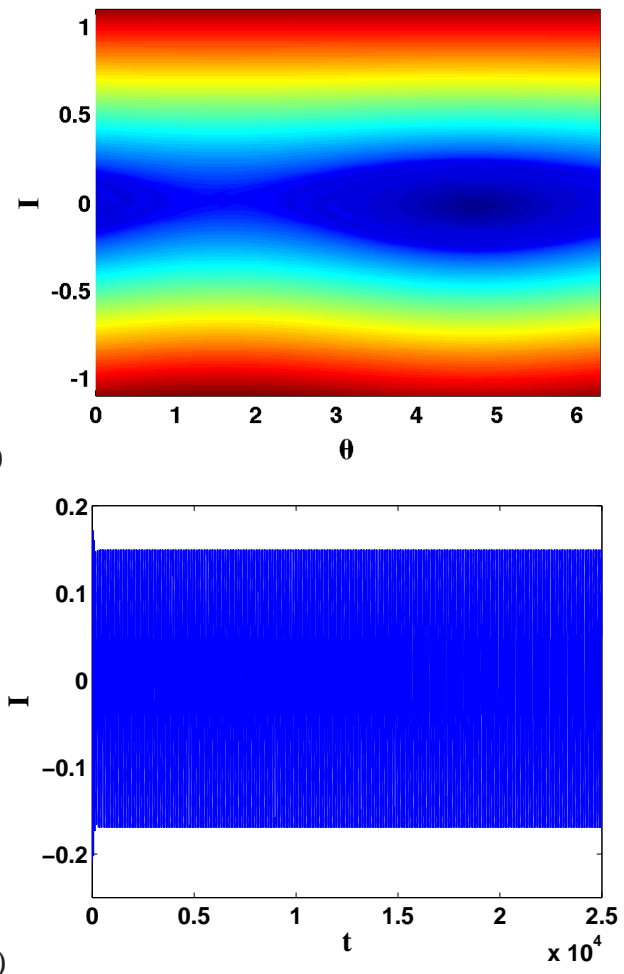

b)

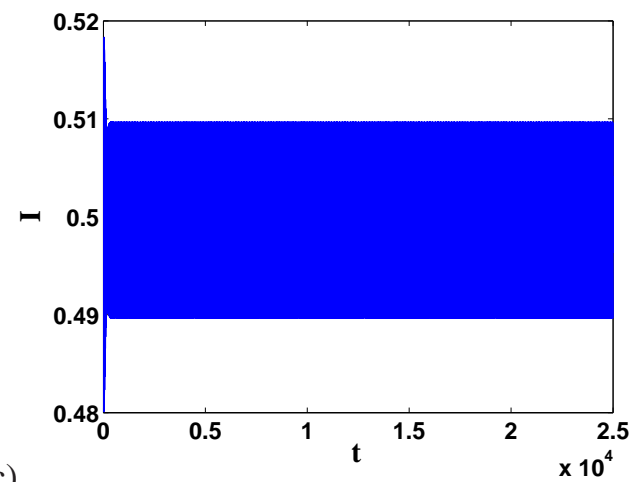

c)

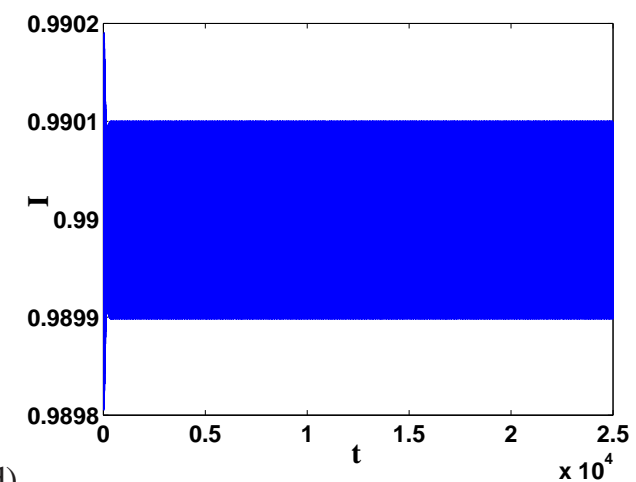

e)

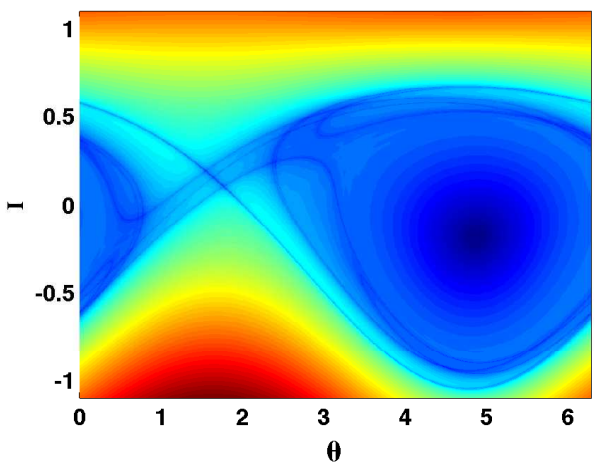

f)
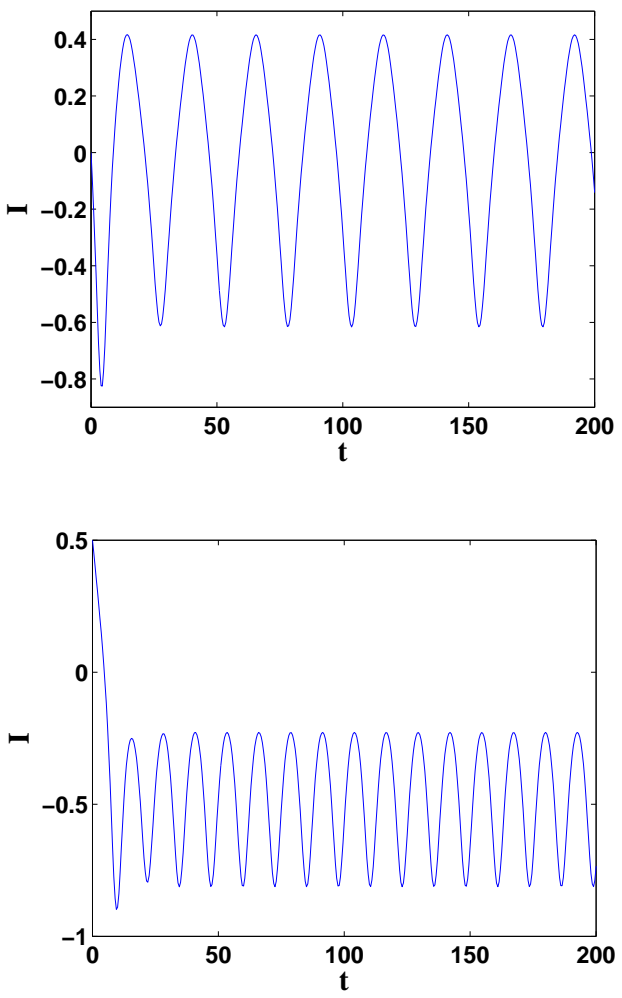

g)

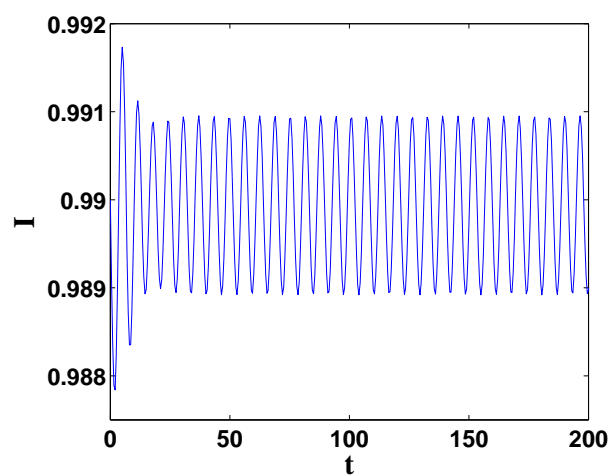

Fig. 4. Results obtained for the time dependence given by Eq. (36). The first column is for $\varepsilon=0.01, \sqrt{\varepsilon}=0.1$. (a) Lagrangian descriptor evaluated in the domain $[0,2 \pi] \times[-1.1,1.1]$ at $t=0$ for $\tau=160 ;(\mathbf{b})$, (c), and (d) show the $I$ component of the trajectory as a function of time for the following initial conditions: (b) $\theta=0, I=0$; (c) $\theta=0, I=0.5$; (d) $\theta=0, I=0.99$. The second column is for $\varepsilon=0.1$, $\sqrt{\varepsilon}=0.31623$. (e) Lagrangian descriptor evaluated in the domain $[0,2 \pi] \times[-1.1,1.1]$ at $t=0$ for $\tau=160$; (f), (g), and (h) show the $I$ component of the trajectory as a function of time for the following initial conditions: (f) $\theta=0, I=0$; $(\mathbf{g}) \theta=0, I=0.5$; (h) $\theta=0, I=0.99$. 
a)
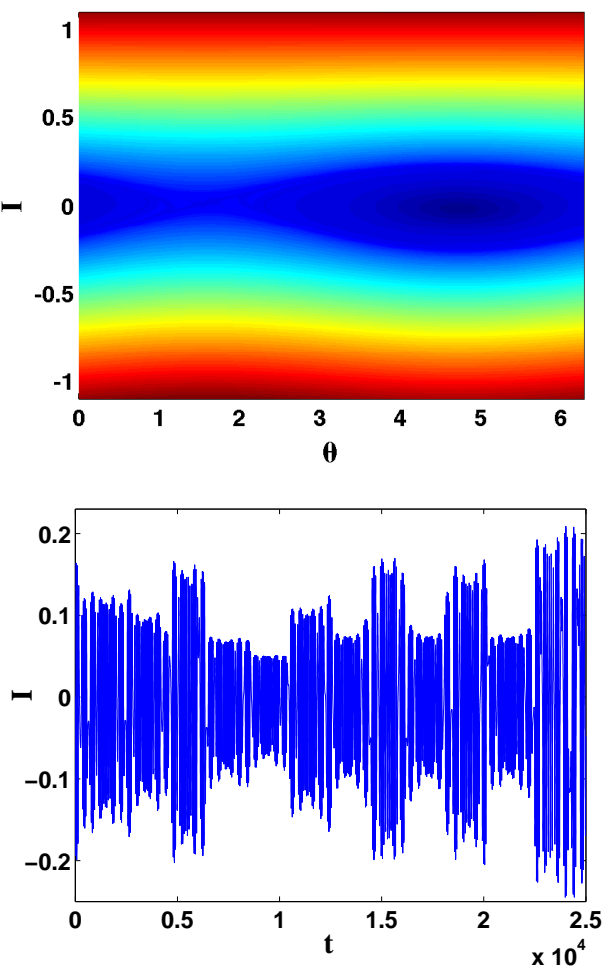

b)

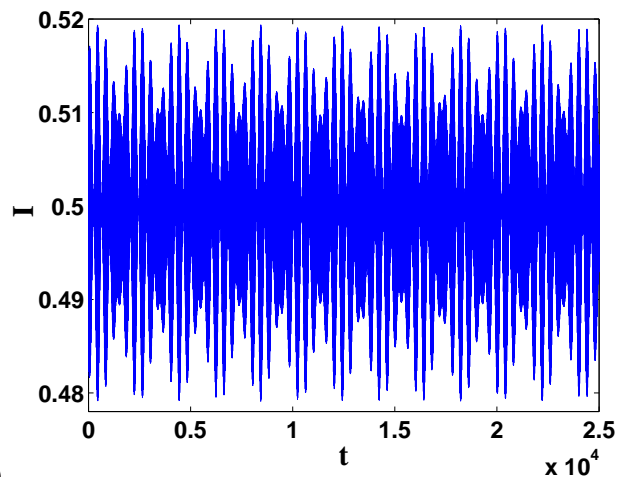

c)

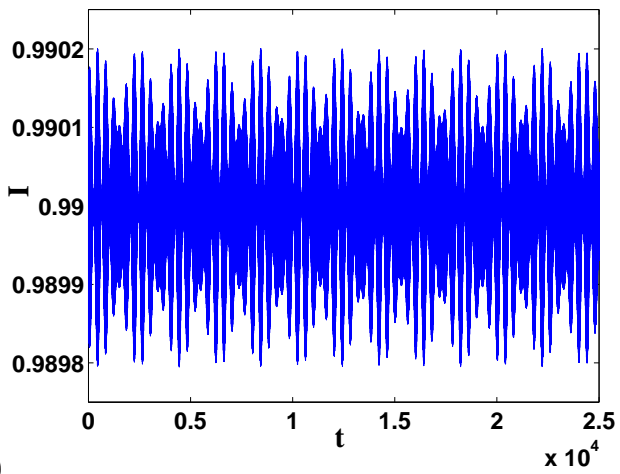

e)
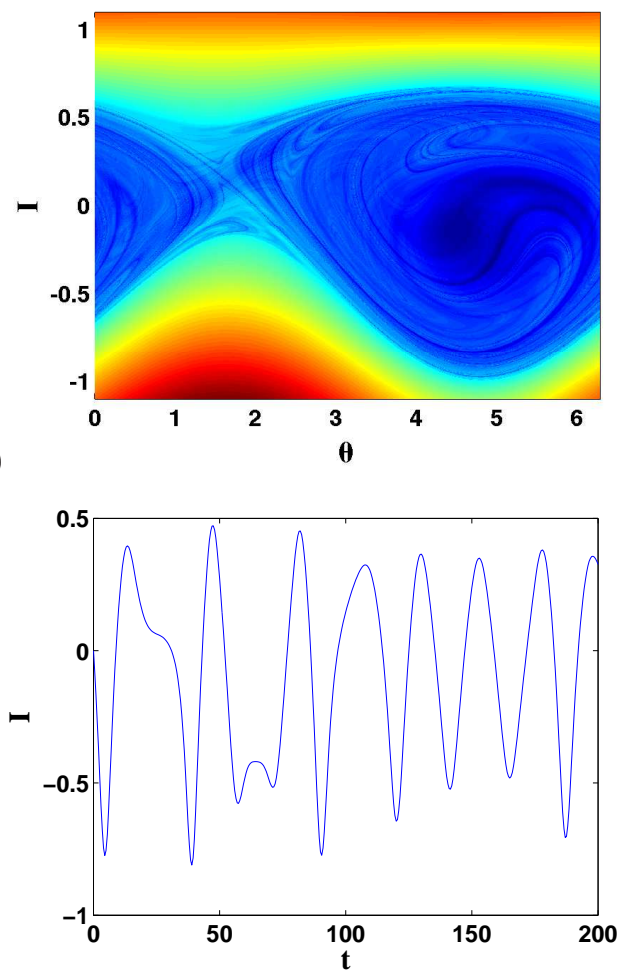

f)

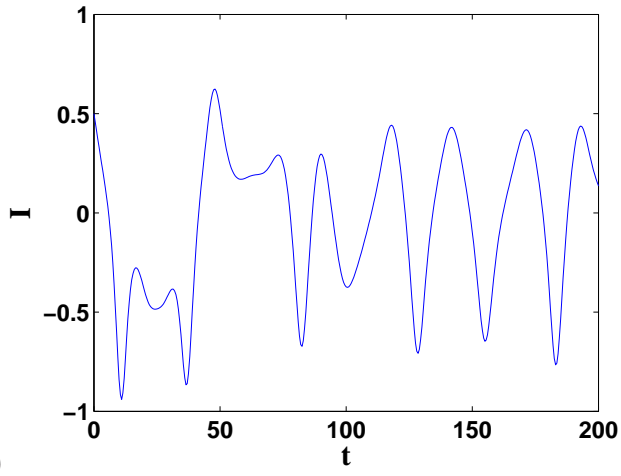

g)

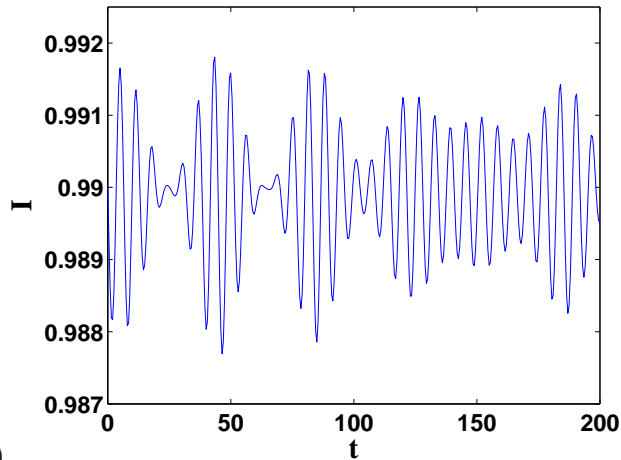

Fig. 5. Results obtained for the time dependence given by Eq. (37). The first column is for $\varepsilon=0.01, \sqrt{\epsilon}=0.1$. (a) Lagrangian descriptor evaluated in the domain $[0,2 \pi] \times[-1.1,1.1]$ at $t=0$ for $\tau=160 ;(\mathbf{b}),(\mathbf{c})$, and (d) show the $I$ component of the trajectory as a function of time for the following initial conditions: (b) $\theta=0, I=0$; (c) $\theta=0, I=0.5$; (d) $\theta=0, I=0.99$. The second column is for $\varepsilon=0.1$, $\sqrt{\varepsilon}=0.31623$. (e) Lagrangian descriptor evaluated in the domain $[0,2 \pi] \times[-1.1,1.1]$ at $t=0$ for $\tau=160$; (f), (g), and (h) show the $I$ component of the trajectory as a function of time for the following initial conditions: (f) $\theta=0, I=0 ;(\mathbf{g}) \theta=0, I=0.5$; (h) $\theta=0, I=0.99$. 


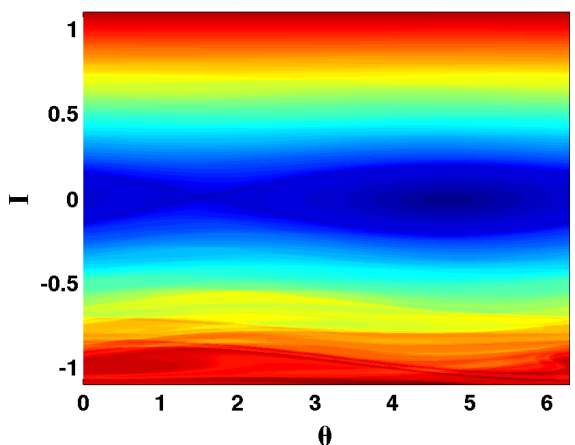

a)

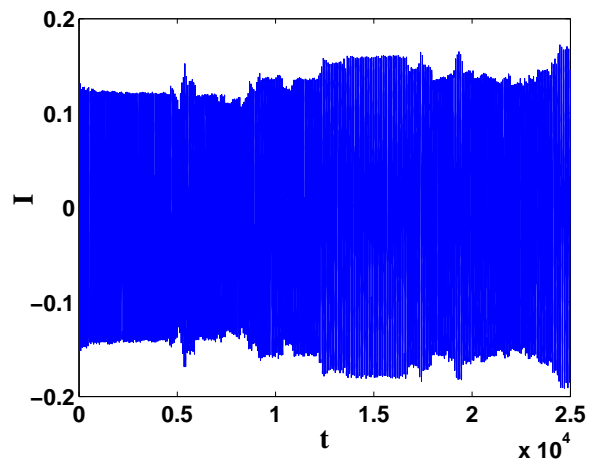

b)

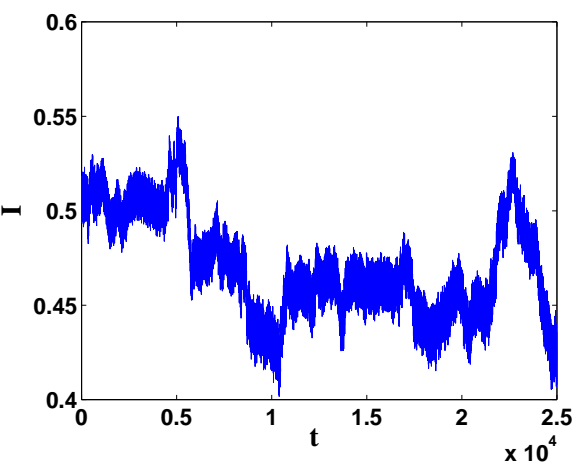

c)

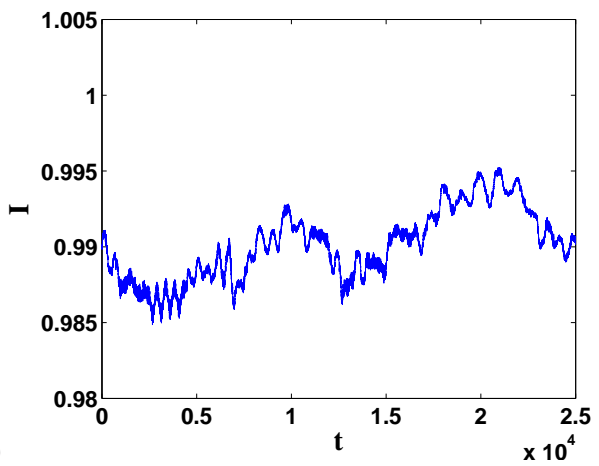

e)

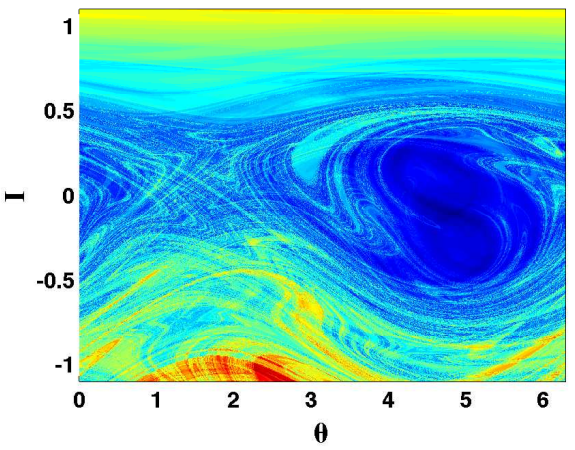

f)

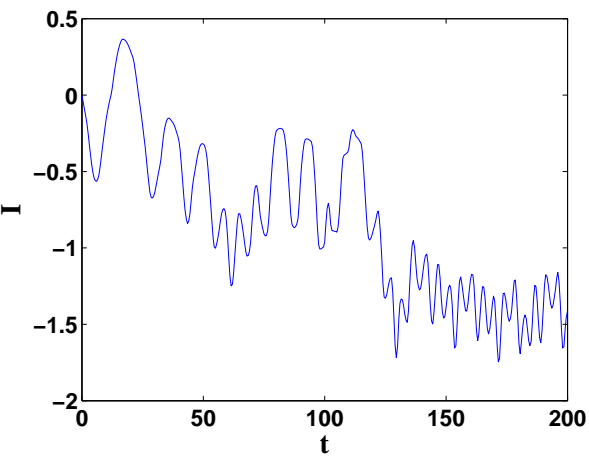

g)
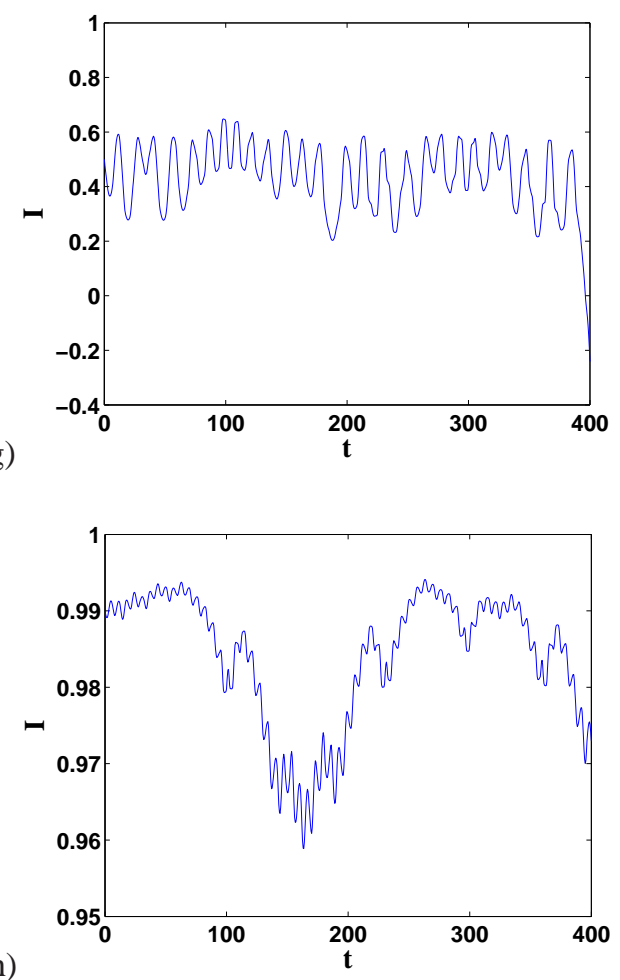

Fig. 6. Results obtained for the time dependence given by the aperiodic forcing obtained from the chaotic output of a differential equation. The first column is for $\varepsilon=0.01, \sqrt{\varepsilon}=0.1$. (a) Lagrangian descriptor evaluated in the domain $[0,2 \pi] \times[-1.1,1.1]$ at $t=0 ;(\mathbf{b}),(\mathbf{c})$, and (d) show the $I$ component of the trajectory as a function of time for the following initial conditions: (b) $\theta=0, I=0$; (c) $\theta=0, I=0.5$; (d) $\theta=0, I=0.99$. The second column is for $\varepsilon=0.1, \sqrt{\varepsilon}=0.31623$. (e) Lagrangian descriptor evaluated in the domain $[0,2 \pi] \times[-1.1,1.1]$ at $t=0$; (f), (g), and (h) show the $I$ component of the trajectory as a function of time for the following initial conditions: (f) $\theta=0, I=0$; (g) $\theta=0, I=0.5$; (h) $\theta=0, I=0.99$. 
In the second column similar agreement with the Nekhoroshev estimates is shown for the trajectories in Fig. $4 \mathrm{f}$ and $\mathrm{h}$. In these figures the confinement is of order $S=c 0.3$ (with $c$ a constant of $O(1))$ for a time of at least $T=25$. The confinement for Fig. $4 \mathrm{~g}$ with initial condition $(I=0.5$ and $\theta=0)$ does not satisfy the Nekhoroshev estimates, since the trajectory rapidly evolves away from the initial condition more than a distance $S$ in time $T<25$. The perturbation size is too large for this initial condition.

An understanding of how the constants, including perturbation size, affect the estimates of confinement distance and stability time requires detailed estimates of the sizes of these constants, taking into account explicit phase space structures, i.e. resonances. Some results along these lines have been obtained in the time-independent case in Benettin and Gallavotti (1986) and Lochak (1992). Similar results have yet to be obtained for the time-dependent case (where the time dependence is aperiodic), but our simulations indicate that this would be a fruitful direction for further research.

Figure 5 shows results for the time dependence (Eq. 37). As before, the first column shows the results for $\varepsilon=0.01$ and the second for $\varepsilon=0.1$. The agreement is as expected in the first column and in the second one the confinement is again not satisfied for the initial condition $(I=0.5$ and $\theta=0)$, as displayed in Fig. 5g.

For an aperiodically time-dependent forcing obtained from a chaotic trajectory, as illustrated in Fig. 3e, the results are shown in Fig. 6. In the first column the results for $\varepsilon=$ 0.01 are in good agreement with what is expected from the Nekhoroshev theorem. In the second column the quality of the results for $\varepsilon=0.1$ depends on the initial condition. The trajectory shown in Fig. 6f satisfies the confinement estimate for $T<25$, and shortly after that the trajectory no longer obeys the estimate. Figure $6 \mathrm{~g}$ confirms that the bound beyond $T \sim 400$ is no longer satisfied by showing a rapid distancing at this point. This is consistent with the estimations obtained from the theorem. Surprisingly for the aperiodic case, the size perturbation $\varepsilon=0.1$ for the initial condition $(I=0.5$ and $\theta=0$ ) has not been too large as it has been in the previous considered time dependencies. Finally, the initial condition in Fig. 6h displays a confinement that is compatible with the estimates of the theorem. Although not shown, the confinement is maintained up to $T \sim 1500$.

It is notable that for both $\varepsilon=0.1$ and $\varepsilon=0.01$ the action variables of trajectories initialised near $I=1$ change very little during the course of their evolution for the three types of forcing considered. Recall that $I=1$ is an invariant torus even for $\varepsilon \neq 0$. This result is reminiscent of results on the "stickiness" of invariant tori in time-independent systems; see Perry and Wiggins (1994); Morbidelli and Giorgilli $(1995 a, b)$. There are no such "stickiness results" for timedependent systems (for any time dependence), so this result might serve as a motivation to seek such results.

\section{Conclusions and outlook}

In this paper we have considered fluid transport in twodimensional flows from the dynamical systems point of view, with the focus on elliptic behaviour and aperiodic and finite time dependence. We have given an overview of previous work on general nonautonomous and finite time vector fields with the purpose of bringing to the attention of those working on fluid transport from the dynamical systems point of view a body of work that is very relevant, but appears not to be so well known. We then focused on the KolmogorovArnold-Moser (KAM) theorem and the Nekhoroshev theorems. While there is no finite time or aperiodically timedependent version of the KAM theorem, the Nekhoroshev theorem, by its very nature, is a finite time result, but for a "very long" (i.e. exponentially long with respect to the size of the perturbation) time interval. In this sense it provides a rigorous description of the notion of "nearly invariant tori" over a finite time interval. Moreover, there is an aperiodically time-dependent version of the Nekhoroshev theorem due to Giorgilli and Zehnder (1992) (recently refined by Bounemoura (2013) and Fortunati and Wiggins (2013)) which is directly relevant to fluid transport problems. We give a detailed discussion of issues associated with the applicability of the KAM and Nekhoroshev theorems in specific flows. Finally, we consider a specific example where we show that the results of the Nekhoroshev theorem hold. We note that the general aperiodic time dependence in this particular version of Nekhoroshev's theorem is "slow" (in a sense that is made clear in the statement of the theorem). Work is underway to generalise this to more general aperiodic time dependence.

Acknowledgement. S. Wiggins would like to acknowledge useful e-mail exchanges with Antonio Giorgilli. The research of S. Wiggins was supported by ONR grant no. N00014-01-1-0769. The research of A. M. Mancho is supported by MINECO under grant MTM2011-26696. S. Wiggins and A. M. Mancho acknowledge support from MINECO: ICMAT Severo Ochoa project SEV-20110087.

Edited by: J. Duan

Reviewed by: two anonymous referees

\section{References}

Acrivos, A., Aref, H., and Ottino, J. M. (Eds.): Symposium on Fluid Mechanics of Stirring and Mixing, Phys. Fluids A, Part 2, 723 1469, 1991.

Aref, H.: Stirring by chaotic advection, J. Fluid Mech., 143, 1-21, 1984.

Aref, H.: The development of chaotic advection, Phys. Fluids, 14, 1315-1325, 2002. 
Aref, H. and El Naschie, M. S. (Eds.): Chaos Applied to Fluid Mixing, Chaos, Solitons, and Fractals, 4, 745-1116, 1994.

Arnold, V. I.: Proof of A. N. Kolmogorov's theorem on the preservation of quasiperodic motions under small perturbations of the Hamiltonian, Russ. Math. Surveys, 18, 9-36, 1963.

Arnold, V. I.: Mathematical Methods of Classical Mechanics, Springer-Verlag, New York, Heidelberg, Berlin, 1978.

Arnold, V. I., Kozlov, V. V., and Neishtadt, A. I.: Mathematical Aspects of Classical and Celestial Mechanics, Dynamical Systems, Vol. III, Springer-Verlag, New York, Heidelberg, Berlin, 1988.

Babiano, A., Provenzale, A., and Vulpiani, A. (Eds.): Chaotic Advection, Tracer Dynamics, and Turbulent Dispersion. Proceedings of the NATO Advanced Research Workshop and EGS Topical Workshop on Chaotic Advection, Conference Centre Sereno di Gavo, Italy, 24-28 May 1993, Physica D, Vol. 76, 1994.

Balibrea, F., Caraballo, T., Kloeden, P. E., and Valero, J.: Recent develpments in dynamical systems: Three perspectives, Int. J. Bif. Chaos, 20, 2591-2636, 2010.

Batchelor, G. K.: An Introduction to Fluid Dynamics, Cambridge University Press, Cambridge, 1967.

Benettin, G. and Gallavotti, G.: Stability of motions near resonances in quasi-integrable Hamiltonian systems, J. Stat. Phys., 44, $293-$ $338,1986$.

Berger, A.: On finite time hyperbolicity, Comm. Pure App. Anal., 10, 963-981, 2011.

Berger, A., Son, D. T., and Siegmund, S.: Nonautonomous finitetime dynamics, Discrete Cont. Dyn.-B, 9, 463-492, 2008.

Berger, A., Doan, T. S., and Siegmund, S.: A definition of spectrum for differential equations on finite time, J. Diff. Eq., 246, 10981118, 2009.

Beron-Vera, F. J., Olascoaga, M. J., Brown, M. G., Kocak, H., and Rypina, I.: Invariant-tori-like Lagrangian coherent structures in geophysical flows, Chaos, 20, 017514, doi:10.1063/1.3271342, 2010.

Bounemoura, A.: Effective stability for slow time-dependent nearintegrable Hamiltonians and application, C. R. Math. Acad. Sci. Paris, 351, 673-676, 2013.

Bower, A. S.: A simple kinematic mechanism for mixing fluid parcels across a meandering jet, J. Phys. Oceanogr., 21, 173-180, 1991.

Branicki, M. and Wiggins, S.: Finite-time Lagrangian transport analysis: stable and unstable manifolds of hyperbolic trajectories and finite-time Lyapunov exponents, Nonlin. Processes Geophys., 17, 1-36, doi:10.5194/npg-17-1-2010, 2010.

Broer, H. W., Huitema, G. B., and Sevryuk, M. B.: Quasi-Periodic Motions in Families of Dynamical Systems, Lecture Notes in Mathematics, Vol. 1645, Springer-Verlag, New York, Heidelberg, Berlin, 1996.

Brown, G. L. and Roshko, A.: Density effect and large structure in turbulent mixing layers, J. Fluid Mech., 64, 775-816, 1974.

Buesseler, K., Jayne, S. R., Fisher, N. S., Rypina, I. I., Baumann, H., Baumann, Z., Breier, C. F., Douglass, E. M., George, J., Macdonald, A. M., Miyamoto, H., Nishikawa, J., Pike, S. M., and Yoshida, S.: Fukushima-derived radionuclides in the ocean and biota off japan, Proc. Natl. Acad. Sci., 109, 5984-5988, 2012.

Celletti, A. and Chierchia, L.: KAM stability and celestial mechanics, Mem. Am. Mathe. Soc., 187, no. 878, 2007.

Chierchia, L. and Mather, J. N.: Kolmogorov-Arnold-Moser theory, Scholarpedia, 5, 2123, doi:10.4249/scholarpedia.2123, 2010.
Chierchia, L. and Pinzari, G.: The planetary N-body problem: symplectic foliation, reductions, and invariant tori, Invent. Math., 186, 1-77, 2011.

Chow, S. N., Lin, X. B., and Palmer, K.: A shadowing lemma with applications to semilinear parabolic equations, SIAM J. Math. Anal., 20, 547-557, 1989.

Coddington, E. A. and Levinson, N.: Theory of Ordinary Differential Equations, McGraw-Hill, New York, 1955.

Coulliette, C. and Wiggins, S.: Intergyre transport in a wind-driven, quasigeostrophic double gyre: An application of lobe dynamics, Nonlin. Processes Geophys., 8, 69-94, doi:10.5194/npg-869-2001, 2001.

Dafermos, C. M.: An invariance principle for compact processes, J. Diff. Eq., 9, 239-252, 1971.

Danckwerts, P. V.: The definition and measurement of some characteristics of mixtures, Appl. Sci. Res., A3, 279-296, 1952.

Danckwerts, P. V.: Theory of mixtures and mixing, Research, 6, 355-361, 1953.

de Blasi, F. S. and Schinas, J.: On the stable manifold theorem for discrete time dependent processes in banach spaces, Bull. London Math. Soc., 5, 275-282, 1973.

de la Cámara, A., Mancho, A. M., Ide, K., Serrano, E., and Mechoso, C.: Routes of transport across the Antarctic polar vortex in the southern spring, J. Atmos. Sci., 69, 753-767, 2012.

de la Cámara, A., Mechoso, R., Mancho, A. M., Serrano, E., and Ide, K.: Quasi-horizontal transport within the antarctic polar night vortex: Rossby wave breaking evidence and lagrangian structures, J. Atmos. Sci., 70, 2982-3001, 2013.

de la Llave, R., González, A., Jorba, A., and Villanueva, J.: KAM theory without action-angle variables, Nonlinearity, 18, 855895, 2005.

Delshams, A. and Gutierrez, P.: Effective stability and KAM theory, J. Diff. Eq., 128, 415-490, 1996.

Doan, T. S., Palmer, K., and Siegmund, S.: Transient spectral theory, stable and unstable cones and Gershgorin's theorem for finitetime differential equations, J. Diff. Eq., 250, 4177-4199, 2011.

Dorato, P.: An overview of finite-time stability, in: Current Trends in Nonlinear Systems and Control: In Honor of Petar Kokotovic and Turi Nicosia, edited by: Menini, L., Zaccarian, L., and Abdallah, C. T., Systems and Control-Foundations and Applications, 185194, Birkhauser, Boston, 2006.

Duan, J. Q. and Wiggins, S.: Fluid exchange across a meandering jet with quasi-periodic time variability, J. Phys. Oceanogr., 26, 1176-1188, 1996.

Duan, J. and Wiggins, S.: Lagrangian transport and chaos in the near wake of the flow around an obstacle: a numerical implementation of lobe dynamics, Nonlin. Processes Geophys., 4, 125-136, doi:10.5194/npg-4-125-1997, 1997.

Duc, L. H. and Siegmund, S.: Hyperbolicity and invariant manifolds for planar nonautonomous systems on finite time intervals, Int. J. Bif. Chaos, 18, 641-674, 2008.

Duc, L. H. and Siegmund, S.: Existence of finite-time hyperbolic trajectories for planar hamiltonian flows, J. Dyn. Diff. Eq., 23, 475-494, 2011.

Eckart, C.: An analysis of the stirring and mixing processes in incompressible fluids, J. Mar. Res., 7, 265-275, 1948.

Fenichel, N.: Hyperbolicity and exponential dichotomy for dynamical systems, in: Dynamics Reported, edited by: Jones, C. K. 
R. T., Kirchgraber, U., and Walther, H. O., Vol. 5, 1-25, SpringerVerlag, New York, Heidelberg, Berlin, 1991.

Fortunati, A. and Wiggins, S.: Normal form and Nekhoroshev stability for nearly-integrable Hamiltonian systems with unconditionally slow aperiodic time dependence., available at: http: //arxiv.org/abs/1311.6290, 2013.

Giorgilli, A.: Quantitative methods in classical perturbation theory, in: From Newton to chaos: modern techniques for understanding and coping with chaos in N-body dynamical systems, edited by: Roy, A. E. and Steves, B. D., 21-38, New York, Plenum Press, 1995.

Giorgilli, A.: Notes on exponential stability of Hamiltonian systems, in: Dynamical Systems. Part I. Hamiltonian Systems and Celestial Mechanics, Pisa, Centro di Recerca Matematica Ennio De Giorgi, Scuola Normale Superiore, 2002.

Giorgilli, A. and Zehnder, E.: Exponential stability for time dependent potentials, Z. angew. Math. Phys. (ZAMP), 43, 827-855, 1992.

Giorgilli, A., Locatelli, U., and Sansottera, M.: Kolmogorov and Nekhoroshev theory for the problem of three bodies, Cel. Mech. Dyn. Astron., 104, 159-173, 2009.

Haller, G.: Finding finite-time invariant manifolds in twodimensional velocity fields, Chaos, 10, 99-108, 2000.

Haller, G. and Poje, A.: Finite time transport in aperiodic flows, Physica D, 119, 352-380, 1998.

Irwin, M. C.: Hyperbolic time dependent processes, Bull. London Math. Soc., 5, 209-217, 1973.

Jones, C. K. R. T. and Winkler, S.: Invariant manifolds and Lagrangian dynamics in the ocean and atmosphere, in: Handbook of dynamical systems, 55-92, North-Holland, Amsterdam, 2002.

Jorba, A. and Simo, C.: On quasiperiodic perturbations of elliptic equilibrium points, SIAM J. Math. Anal., 27, 1704-1737, 1996.

Katok, A. and Hasselblatt, B.: Introduction to the Modern Theory of Dynamical Systems, Cambridge University Press, Cambridge, 1995.

Kloeden, P. E. and Poetzsche, C.: Non-autonomous difference equations and discrete dynamical systems. J. Difference Eq. Appl., 17, 129-130, 2011.

Kloeden, P. and Schmalfuss, B.: Nonautonomous systems, cocycle attractors, and variable time-step discretization, Num. Algor., 14, 141-152, 1997.

Kolmogorov, A. N.: On conservation of conditionally periodic motions under small perturbations of the Hamiltonian, Dokl. Akad. Nauk. USSR, 98, 527-530, 1954.

Langa, J. A., Robinson, J. C., and Suàrez, A.: Stability, instability, and bifurcation phenomena in non-autonomous differential equations, Nonlinearity, 15, 887-903, 2002.

Lerman, L. and Silnikov, L.: Homoclinical structures in nonautonomous systems: Nonautonomous chaos, Chaos, 2, 447-454, 1992.

Littlewood, J. E.: The Lagrange configuration in celestial mechanics, Proc. London Math. Soc., 9, 525-543, 1959a.

Littlewood, J. E.: On the equilateral configuration of the restricted problem of three bodies, Proc. London Math. Soc., 9, 343-372, 1959b.

Lochak, P.: Canonical perturbation theory via simultaneous approximation, Russ. Math. Surveys, 47, 57-133, 1992.

Lochak, P.: Hamiltonian perturbation theory: periodic orbits, resonances, and intermittency, Nonlinearity, 6, 885-904, 1993.
Lochak, P. and Porzio, A.: A realistic exponential estimate for a paradigm Hamiltonian, Annales de l'I. H. P, section A, 51, 199219, 1989.

Lu, K. and Wang, Q.: Chaos in differential equations driven by a nonautonomous force, Nonlinearity, 23, 2935-2973, 2010.

Lu, K. and Wang, Q.: Chaotic behavior in differential equations driven by a Brownian motion, J. Diff. Eq., 251, 2853-2895, 2011.

Malhotra, N. and Wiggins, S.: Geometric structures, lobe dynamics, and Lagrangian transport in flows with aperiodic timedependence, with applications to Rossby wave flow, J. Nonlinear Science, 8, 401-456, 1998.

Mancho, A. M., Small, D., and Wiggins, S.: Computation of hyperbolic trajectories and their stable and unstable manifolds for oceanographic flows represented as data sets, Nonlin. Processes Geophys., 11, 17-33, doi:10.5194/npg-11-17-2004, 2004.

Mancho, A. M., Small, D., and Wiggins, S.: A tutorial on dynamical systems concepts applied to Lagrangian transport in oceanic flows defined as finite time data sets: Theoretical and computational issues, Phys. Reports, 437, 55-124, 2006.

Mancho, A. M., Wiggins, S., Curbelo, J., and Mendoza, C.: Lagrangian descriptors: A method for revealing phase space structures of general time dependent dynamical systems, Communic. Nonlin. Sci. Num. Simul., 18, 3530-3557, 2013.

Meiss, J. D.: Visual explorations of dynamics: The standard map, PRAMANA-J. Phys., 70, 965-988, 2008.

Mendoza, C. and Mancho, A. M.: The hidden geometry of ocean flows, Phys. Rev. Lett., 105, 038501, doi:10.1103/PhysRevLett.105.038501, 2010.

Mendoza, C. and Mancho, A. M.: Review Article: "The Lagrangian description of aperiodic flows: a case study of the Kuroshio Current", Nonlin. Processes Geophys., 19, 449-472, doi:10.5194/npg-19-449-2012, 2012.

Meyer, K. R. and Sell, G. R.: Melnikov transforms, bernoulli bundles, and almost periodic perturbations, Trans. Amer. Math. Soc., 314, 63-105, 1989.

Meyer, K. R. and Zhang, X.: Stability of skew dynamical systems, J. Diff. Eq., 132, 66-86, 1996.

Miller, P. D., Jones, C. K. R. T., Rogerson, A. M., and Pratt, L. J.: Quantifying transport in numerically generated velocity fields, Physica D, 110, 105-122, 1997.

Miller, R. K.: Almost periodic differential equations as dynamical systems with applications to the existence of almost periodic solutions, J. Diff. Eq., 1, 337-395, 1965.

Morbidelli, A. and Giorgilli, A.: On an connection between KAM and Nekhorshev's theorems. Physica D, 86, 514-516, 1995a.

Morbidelli, A. and Giorgilli, A.: Superexponential stability of KAM tori, J. Stat. Phys., 78, 1607-1617, 1995b.

Moser, J.: Stabilitätsverhalten kanonischer Differentialgleichungssysteme, Nachrichten der Akademie der Wissenschaften in Göttingen. II. Mathematisch-Physikalische Klasse, 1955, 87-120, 1955.

Moser, J.: On invariant curves of an area preserving mappings of an annulus, Nachr. Akad. Wiss. Gött., II. Math.-Phys. K1., 1-20, 1962.

Nekhoroshev, N. N.: An exponential estimate on the time of stabilty of nearly-integrable Hamiltonian systems, Russ. Math. Surveys, 32, 1-65, 1977. 
Ottino, J.: The Kinematics of Mixing: Stretching, Chaos, and Transport, Cambridge University Press, Cambridge, 1989a.

Ottino, J. M.: The mixing of fluids, Scientif. Am., 260, 56-67, 1989b.

Ottino, J. M., Jana, S. C., and Chakravarthy, V. S.: From Reynolds stretching and folding to mixing studies using horseshoe maps, Phys. Fluids, 6, 685-699, 1994.

Perry, A. D. and Wiggins, S.: KAM tori are very sticky: rigorous lower bounds on the time to move away from an invariant Lagrangian torus with linear flow tori are very, Physica D, 71, 102121, 1994

Pesin, Y.: Lectures on Partial Hyperbolicity and Stable Ergodicity, Zurich Lectures in Advanced Mathematics, European Mathematical Society, 2004.

Poetzsche, C.: Geometric Theory of Discrete Nonautonomous Dynamical Systems, Lecture Notes in Mathematics, Vol. 2002, Springer-Verlag, Berlin, 2010a.

Poetzsche, C.: Nonautonomous bifurcation of bounded solutions I, A Lyapunov-Schmidt approach, Discrete and continuous dynamical systems-series B, 14, 739-776, 2010b.

Poetzsche, C.: Nonautonomous bifurcation of bounded solutions II, A shovel bifurcation pattern, Discrete Contin. Dyn. Syst., 31, 941-973, 2011a.

Poetzsche, C.: Persistence and imperfection of nonautonomous bifurcation patterns, J. Diff. Eq., 250, 3874-3906, 2011 b.

Pöschel, J.: Nekhoroshev estimates for quasi-convex Hamiltonian systems, Math. Z., 213, 187-216, 1993.

Pöschel, J.: A lecture on the classical KAM theorem, Proc. Symp. Pure Math., 69, 707-732, 2001.

Rasmussen, M.: Towards a bifurcation theory for nonautonomous difference equations. J. Difference Eq. Appl., 12, 297-312, 2006.

Reynolds, O.: Study of fluid motion by means of coloured bands, Nature, 50, 161-164, 1894.

Rogerson, A. M., Miller, P. D., Pratt, L. J., and Jones, C. K. R. T.: Lagrangian motion and fluid exchange in a barotropic meandering jet, J. Phys. Oceanogr., 29, 2635-2655, 1999.

Samelson, R. M.: Fluid exchange across a meandering jet, J. Phys. Oceanogr., 22, 431-440, 1992.

Samelson, R. and Wiggins, S.: Lagrangian Transport in Geophysical Jets and Waves: The Dynamical Systems Approach, SpringerVerlag, New York, 2006.
Scheurle, J.: Chaotic solutions of systems with almost periodic forcing, Z. Angew. Math. Phys., 37, 12-26, 1986.

Sell, G. R.: Nonautonomous differential equationa and topological dynamics I, The basic theory, Trans. Amer. Math. Soc., 127, 241-262, 1967a.

Sell, G. R.: Nonautonomous differential equationa and topological dynamics II, Limiting equations, Trans. Amer. Math. Soc., 127, 263-283, 1967b.

Sell, G. R.: Topological Dynamics and Differential Equations. Van Nostrand-Reinhold, London, 1971.

Sevryuk, M. B.: The classical KAM theory at the dawn of the twenty-first century, Moscow Math. J., 3, 1113-1144, 2003.

Sevryuk, M. B.: Invariant tori in quasiperiodic non-autonomous dynamical systems via Herman's method, Discrete Contin. Dyn. Syst., 18, 569-595, 2007.

Siegmund, S.: Normal forms for nonautonomous differential equations, J. Diff. Eq., 178, 541-573, 2002.

Stoffer, D.: Transversal homoclinic points and hyperbolic sets for non-autonomous maps i, J. Appl. Math. and Phys. (ZAMP), 39, 518-549, 1988a.

Stoffer, D.: Transversal homoclinic points and hyperbolic sets for non-autonomous maps ii, J. Appl. Math. and Phys. (ZAMP), 39, 783-812, 1988b.

Sturman, R., Ottino, J. M., and Wiggins, S.: The Mathematical Foundations of Mixing, Cambridge Monographs on Applied and Computational Mathematics, Vol. 22, Cambridge University Press, 2006.

Weiss, L. and Infante, E. F.: On the stability of systems defined over a finite time interval, Proc. Nat. Acad. Sci., 54, 44-48, 1965.

Wiggins, S.: Chaotic Transport in Dynamical Systems, SpringerVerlag, New York, 1992.

Wiggins, S.: Chaos in the dynamics generated by sequences of maps, with applications to chaotic advection in flows with aperiodic time dependence, Z. Angew. Math. Phys., 50, 585-616, 1999.

Wiggins, S.: The dynamical systems approach to Lagrangian transport in oceanic flows, Annu. Rev. Fluid Mech., 37, 295-328, 2005.

Wiggins, S. and Ottino, J.: Foundations of chaotic mixing, Phil. Trans. Roy. Soc., 362, 937-970, 2004. 